\title{
Chiral phase transition in the soft-wall model of AdS/QCD
}

\author{
Kaddour Chelabi, ${ }^{a, b, c}$ Zhen Fang, ${ }^{a, b}$ Mei Huang, ${ }^{d, e}$ Danning $\mathbf{L i}^{a}$ and Yue-Liang Wu $\mathbf{W u}^{a, b}$ \\ ${ }^{a}$ State Key Laboratory of Theoretical Physics, Institute of Theoretical Physics, \\ Chinese Academy of Sciences, \\ Beijing 100190, P.R. China \\ ${ }^{b}$ University of Chinese Academy of Sciences (UCAS), \\ Beijing 100049, P.R. China \\ ${ }^{c}$ Laboratory of Particle Physics and Statistical Physics, Ecole Normale Superieure-Kouba, \\ B.P. 92,16050, Vieux-Kouba, Algiers, Algeria \\ ${ }^{d}$ Institute of High Energy Physics, Chinese Academy of Sciences, \\ Beijing 100049, P.R. China \\ ${ }^{e}$ Theoretical Physics Center for Science Facilities, Chinese Academy of Sciences, \\ Beijing 100049, P.R. China \\ E-mail: kaddour.chelabi@itp.ac.cn, fangzhen@itp.ac.cn, \\ huangm@ihep.ac.cn, lidn@itp.ac.cn, ylwu@itp.ac.cn
}

ABSTRACT: We investigate the chiral phase transition in the soft-wall model of AdS/QCD at zero chemical potential for two-flavor and three-flavor cases, respectively. We show that there is no spontaneous chiral symmetry breaking in the original soft-wall model. After detailed analysis, we find that in order to realize chiral symmetry breaking and restoration, both profiles for the scalar potential and the dilaton field are essential. The scalar potential determines the possible solution structure of the chiral condensate, except the mass term, it takes another quartic term for the two-flavor case, and for the three-flavor case, one has to take into account an extra cubic term due to the t'Hooft determinant interaction. The profile of the dilaton field reflects the gluodynamics, which is negative at a certain ultraviolet scale and approaches positive quadratic behavior at far infrared region. With this set-up, the spontaneous chiral symmetry breaking in the vacuum and its restoration at finite temperature can be realized perfectly. In the two-flavor case, it gives a second order chiral phase transition in the chiral limit, while the transition turns to be a crossover for any finite quark mass. In the case of three-flavor, the phase transition becomes a first order one in the chiral limit, while above sufficient large quark mass it turns to be a crossover again. This scenario agrees exactly with the current understanding on chiral phase transition from lattice QCD and other effective model studies.

KeYwords: Gauge-gravity correspondence, AdS-CFT Correspondence, Phase Diagram of QCD

ARXIV EPRINT: 1512.06493 


\section{Contents}

1 Introduction 1

2 Chiral symmetry breaking in original soft-wall model 4

2.1 General setup 4

2.2 Quark mass and temperature dependence of chiral condensate 6

3 Quartic potential effects $\quad 8$

3.1 Chiral condensate in positive quadratic dilaton background $\quad 10$

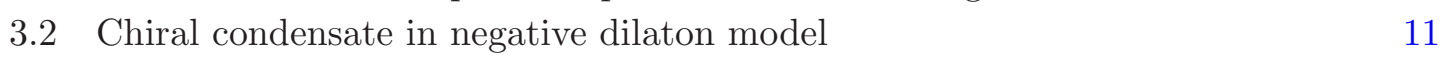

4 Interpolated dilaton: two different scales $\quad \mathbf{1 4}$

$\begin{array}{lll}4.1 & \mathrm{SU}(2) \text { case: } v_{3}=0, v_{4} \neq 0 & 15\end{array}$

$\begin{array}{lll}4.2 & \mathrm{SU}(3) \text { case: } v_{3}, v_{4} \neq 0 & 19\end{array}$

$\begin{array}{lll}5 & \text { Conclusion and discussion } & 21\end{array}$

$\begin{array}{ll}\text { A Numerical method } & 24\end{array}$

\section{Introduction}

The vacuum of Quantum Chromodynamics(QCD), which is well known as the theory of Strong Interactions, is characterized by spontaneous chiral symmetry breaking and color charge confinement. And it is widely believed that at sufficient high temperature and/or density phase transition would happen in the system: chiral symmetry could be restored and color degrees of freedom can be freed. At present, to understand the phase structure of these two phase transitions is an important topic in both non-perturbative QCD study and cosmology [1].

The properties of QCD phase transition depend sensitively on the inertial quantities of the system, such as the number $\left(N_{f}\right)$ of flavors and the mass of quarks $\left(m_{u}, m_{d}\right.$ and $\left.m_{s}\right)$. The chiral phase transition and confinement/deconfinement phase transition are well defined as a true phase transition only in the chiral limit and in the infinite quark mass limit respectively, when the chiral symmetry and $Z_{3}$ centre symmetry are the exact symmetries of QCD. In these cases, chiral condensate $\langle\bar{\psi} \psi\rangle$ and Polyakov loop $\langle L\rangle$ are well defined order parameters for chiral restoration and color charge deconfinement respectively. In the physical quark mass region, there are no known exact symmetries and the phase transitions are widely accepted as a continual transition, called crossover.

Based on theoretical consideration and lattice QCD simulation [2-4], the expected three flavor phase diagram in the quark mass plane can be summarized in the sketch plot (sometimes called "Columbia Plot") shown in figure 1 (taken from [2]). Both near vanishing 


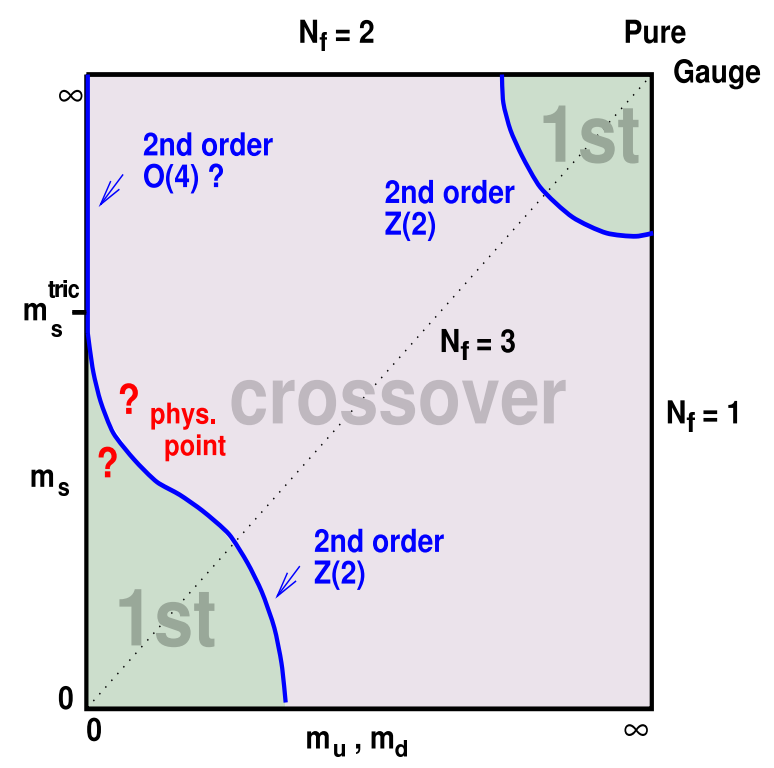

Figure 1. The expected phase diagram in the quark mass $m_{u}=m_{d}, m_{s}$ space (taken from [2]).

current quark mass region $m_{u}=m_{d}=m_{s}=0$ and near the infinite current quark mass region $m_{u}=m_{d}=m_{s}=\infty$, the phase transitions are widely accepted to be of first order, while in the intermediate region it becomes crossover. The boundaries between different regions are second order lines. In this paper, we will first focus on the two light flavor region with $m_{u}=m_{d} \simeq O(\mathrm{MeV})$ and $m_{s}=\infty$ and the SU(3) diagonal line with $m_{u}=m_{d}=m_{s}$. For the $\mathrm{SU}(2)$ case the phase transition starts from a second order one in the chiral limit $m_{u}=m_{d}=0$ (in analogy to $O(4) \sigma$ model [5] noting that $\mathrm{SU}(2)_{L} \times \mathrm{SU}(2)_{R} \simeq O(4)$ ). Then it is expected that even very small quark mass would drive the second order transition to a crossover one, as shown in figure 1. For the $\mathrm{SU}(3)$ case, the phase transition would start from a first order one in chiral limit and turn to crossover one at sufficient large quark mass.

The dominant physics for QCD phase transition is non-perturbative, hence perturbative methods become invalid in this region. Lattice QCD simulations are widely accepted as the most reliable method to study non-perturbative properties of QCD. However, despite the significant improvements which have been done in lattice QCD studies, it is still unable to get full understanding on QCD phase diagram from this ab initial approach. For example, the serious difficulty called sign problem prevents direct lattice simulations of QCD at finite chemical potential. Therefore, it is quite necessary to develop other nonperturbative methods to study the non-perturbative dynamics of QCD. In recent decades, the discovery of the anti-de Sitter/conformal field theory (AdS/CFT) correspondence and the conjecture of the gravity/gauge duality [6-8] provides a new powerful tool to solve the strong coupling problem of gauge theory, and shed lights on the full understanding of QCD phase transitions.

By breaking the conformal symmetry in different ways, many efforts have been made towards more realistic holographical description of QCD in non-perturbative region, such as hadron physics [9-26] and hot/dense QCD matter [27-42], both in top-down approaches 
and in bottom-up approaches (see [43-47] for reviews). For QCD phase transitions, most of the bottom-up studies [48-72] focus only on confinement/deconfinement phase transition. In these studies, in some sense, the geometric phases are distinguished by whether the expectation value of Polyakov Loop $\langle\mathrm{L}\rangle$ calculated in the dual gravity background is vanishing or not. However, lattice simulations $[73,74]$ have shown that the extracted phase transition temperatures from chiral condensate $\langle\bar{\psi} \psi\rangle$ and Polyakov Loop $\langle\mathrm{L}\rangle$ are different for physical quark mass. For chiral restoration in $u, d$ sectors, the transition temperature is around $T_{c}^{\chi,(u, d)}=151 \mathrm{MeV}$ while for confinement/deconfinement the transition temperature is around $T_{c}^{d}=176 \mathrm{MeV}$. Therefore, it is necessary to study the temperature dependent chiral condensate and try to get the information of chiral phase transition in holographic framework.

In bottom-up approaches, hard-wall model [9] and soft-wall model [10] are successful in describing hadron physics. Their extended models [19-26] can describe the hadron spectra and the related quantities in very good accuracy. In these models, the chiral condensate is introduced to realize chiral symmetry breaking at zero temperature. However, unlike in Nambu-Jona-Lasinio(NJL) model [75, 76], the value of the chiral condensate is not self-consistently solved from the models themselves. Instead, it is often taken as a free parameter to fit the hadron spectra at zero temperature. The authors of [77] note that the IR boundary condition may require the dependence of chiral condensate on quark mass and try to solve chiral condensate self-consistently. By introducing black hole background and $\mathrm{U}(1)$ gauge field, they extended the calculation to finite temperature and density, in such region they get the phase diagram for chiral phase transition which agrees with the previous approaches(see e.g. [78]). However, since their studies based on the simplest assumption on the gravity background as the original soft-wall model, the quark mass dependence of the chiral condensate and chiral phase transition is not analysed in their work which is our main case in our paper. Noting that the dilaton profile is in the central place to generate correct quark mass dependence behavior of chiral condensate, we try to study the effects of dilaton profile on the chiral phase transition in [79]. This work is an extension of [79], and we will study the dilaton and scalar potential effects in details.

The paper is organized as follows. In section 2, we explain why in the previous set-up the quark mass dependence behavior is not correct, and why a quartic potential term of the scalar field is necessary. Then in section 3, we study the effects of scalar potential and dilaton profile on chiral condensate, and we show that under the negative quadratic dilaton model and quartic scalar potential the quark-mass dependence behavior of chiral phase transition is realized correctly, though there is massless scalar meson state in this model. In section 4 , to get rid of the massless scalar mode, we propose a dilaton profile negative at certain ultraviolet scale and positive at far infrared scale, and we show that chiral phase transition could be well described in this model. In section 5 , a short summary and discussion are listed. We also describe the numerical process we used to extract chiral condensate in appendix A. 


\section{Chiral symmetry breaking in original soft-wall model}

As we mentioned in the above, soft-wall model provides a start point to study linear confinement and chiral symmetry breaking of QCD in bottom-up approach, and the prediction of meson spectrum in its extended models is in good agreement with the experimental data [19-25]. Therefore it is interesting to investigate the spontaneous chiral symmetry breaking of QCD and its restoration in this model. In this section we will give a brief review of this model and then try to study the temperature dependent behavior of chiral condensate, which is the order parameter of chiral phase transition. It should be noted that the similar study in this section has been studied in [77]. However there the authors have not studied the quark mass dependence of chiral condensate, which is of the main interest of this manuscript.

\subsection{General setup}

In the original paper of soft-wall model [10], the authors promote the $4 \mathrm{D}$ global chiral symmetry $\mathrm{SU}(2)_{L} \times \mathrm{SU}(2)_{R}$ of QCD to $5 \mathrm{D}$, and consider the following action

$$
S=-\int d^{5} x \sqrt{-g} e^{-\Phi} \operatorname{Tr}\left(D_{m} X^{+} D^{m} X+M_{5}^{2} X^{+} X+\frac{1}{4 g_{5}^{2}}\left(F_{L}^{2}+F_{R}^{2}\right)\right),
$$

with $A_{L / R}$ the left/right hand gauge field, $D_{m}$ the covariant derivative defined as $D_{m} X=$ $\partial_{m} X-i A_{m}^{L} X+i X A_{m}^{R}, F_{m n}$ the field strength defined as $F_{m n}=\partial_{m} A_{n}-\partial_{n} A_{m}-i\left[A_{m}, A_{n}\right]$, $g$ the determinant of metric, and $\Phi$ the dilaton field. The mass of the complex scalar field $X M_{5}^{2}$ can be determined as $M_{5}^{2}=-3$ (we take the AdS radius $L=1$ in this work) from the AdS/CFT prescription $M_{5}^{2}=(\Delta-p)(\Delta+p-4)$ [8] by taking $\Delta=3, p=0$.

If the scalar field $X$ gets a non-vanishing vacuum expectation value $X_{0}$, then the $\mathrm{SU}(2)_{L} \times \mathrm{SU}(2)_{R}$ symmetry would be broken, in which the soft-wall model can mimic chiral symmetry breaking of QCD. If one assumes that $m_{u}=m_{d}$, the symmetry should be broken to $\mathrm{SU}(2)$ and $X_{0}$ would have the form $\frac{\chi(z)}{2} I_{2}$. Here $I_{2}$ is the $2 \times 2$ identity matrix and $\chi(z)$ is assumed to depend only on the fifth coordinate $z$. It should be noted that by considering $X$ as an $N_{f} \times N_{f}$ matrix and taking the generators from $\mathrm{SU}\left(N_{f}\right)$, it is easy to extend this model to $\mathrm{SU}\left(N_{f}\right)_{L} \times \mathrm{SU}\left(N_{f}\right)_{R}$ case. The same procedure can be done by replacing $X_{0}=\frac{\chi(z)}{\sqrt{2 N_{f}}} I_{N_{f}}$. Here the factor $\sqrt{2 N_{f}}$ is chosen to keep the kinetic term of $\chi$ canonical. After these assumptions, the effective description of the vacuum expectation value of $X$ in terms of $\chi$ reads

$$
S_{\chi}=-\int d^{5} x \sqrt{-g} e^{-\Phi}\left(\frac{1}{2} g^{z z} \chi^{\prime 2}+\frac{1}{2} M_{5}^{2} \chi^{2}\right),
$$

where' denotes the derivative with respect to $z$.

Under the metric ansatz

$$
d s^{2}=e^{2 A_{s}(z)}\left(-d t^{2}+d z^{2}+d x_{i} d x^{i}\right),
$$

$\chi(z)$ could be solved from the following equation

$$
\chi^{\prime \prime}+\left(3 A_{s}^{\prime}-\Phi^{\prime}\right) \chi^{\prime}+3 e^{2 A_{s}} \chi=0 .
$$


From this equation, the ultraviolet(UV,z $\rightarrow 0$ ) asymptotic behavior could be solved as

$$
\chi(z)=c_{1} z+c_{3} z^{3}+\ldots,
$$

with $c_{1}, c_{3}$ two integral constants of the second order ordinary derivative equation (ODE). From the assumption, $X$ is dual to $\bar{q} q$ operator, so one can related $c_{1}, c_{3}$ to the quark mass and chiral condensate as

$$
c_{1}=m_{q} \zeta, c_{3}=\sigma / \zeta
$$

with the normalization factor $\zeta=\frac{\sqrt{N_{c}}}{2 \pi}$ [80]. Then, the meson spectral in this model can be read from poles in corresponding Green functions, or equivalent by solving the following Schrodinger-like equations (for details, see [10], and also [24, 25])

$$
\begin{aligned}
-s_{n}^{\prime \prime}+V_{s}(z) s_{n} & =m_{n}^{2} s_{n}, \\
-\pi_{n}^{\prime \prime}+V_{\pi, \varphi} \pi_{n} & =m_{n}^{2}\left(\pi_{n}-e^{A_{s}} \chi \varphi_{n}\right), \\
-\varphi_{n}^{\prime \prime}+V_{\varphi} \varphi_{n} & =g_{5}^{2} e^{A_{s}} \chi\left(\pi_{n}-e^{A_{s}} \chi \varphi_{n}\right), \\
-v_{n}^{\prime \prime}+V_{v}(z) v_{n} & =m_{n, v}^{2} v_{n} \\
-a_{n}^{\prime \prime}+V_{a} a_{n} & =m_{n}^{2} a_{n},
\end{aligned}
$$

with the schrodinger-like potentials

$$
\begin{aligned}
V_{s} & =\frac{3 A_{s}^{\prime \prime}-\phi^{\prime \prime}}{2}+\frac{\left(3 A_{s}^{\prime}-\phi^{\prime}\right)^{2}}{4}+3 e^{2 A_{s}} \\
V_{\pi, \varphi} & =\frac{3 A_{s}^{\prime \prime}-\phi^{\prime \prime}+2 \chi^{\prime \prime} / \chi-2 \chi^{\prime 2} / \chi^{2}}{2}+\frac{\left(3 A_{s}^{\prime}-\phi^{\prime}+2 \chi^{\prime} / \chi\right)^{2}}{4}, \\
V_{\varphi} & =\frac{A_{s}^{\prime \prime}-\phi^{\prime \prime}}{2}+\frac{\left(A_{s}^{\prime}-\phi^{\prime}\right)^{2}}{4} \\
V_{v} & =\frac{A_{s}^{\prime \prime}-\phi^{\prime \prime}}{2}+\frac{\left(A_{s}^{\prime}-\phi^{\prime}\right)^{2}}{4} \\
V_{a} & =\frac{A_{s}^{\prime}-\phi^{\prime}}{2}+\frac{\left(A_{s}^{\prime}-\phi^{\prime}\right)^{2}}{4}+g_{5}^{2} e^{2 A_{s}} \chi^{2} .
\end{aligned}
$$

Here, $s_{n}, \pi_{n}, v_{n}, a_{n}$ are the $5 \mathrm{D}$ wave function of scalar, pseudoscalar, vector and axial-vector meson respectively.

In the original model, the authors took the $A d S_{5}$ metric, i.e. $A_{s}=-\log (z)$ in eq. (2.3), and one can check that if $\Phi(z)$ increases as $z^{2}$ in $\operatorname{Infrared(IR)~region}(z \rightarrow \infty)$, the mass square of the highly excited meson states would be proportional to the radial quantum number $n$, i.e. $m_{n}^{2} \propto n$ when $n \gg 1$. Therefore, in the original model, the authors simply took $\Phi \propto z^{2}$ to realize the linear spectral. Furthermore, one can read from eqs. (2.15), (2.16) that if $\chi \neq 0$, the equations of motion are different for vector and axial-vector mesons, which shows the possibility to realize chiral symmetry breaking in this model.

In this section, we have seen that the quadratic dilaton at IR and the non-vanishing scalar $\chi$ are important for linear confinement and chiral symmetry breaking of QCD vacuum respectively. The linear confinement has already been studied widely in the extended 
models of the original soft-wall model, and it turns out that soft-wall model does give success description in this topic. However, the chiral symmetry breaking and its restoration are much less studied in this framework. Therefore, in the next section we will focus on this topic.

\subsection{Quark mass and temperature dependence of chiral condensate}

In this section, we would like to study the quark mass and temperature dependence of chiral condensate in the original soft-wall model. The main purpose is to check whether the symmetry breaking is a spontaneous one as that in real QCD or not.

We start from the soft-wall action eq. (2.2), and under the usual metric ansatz at finite temperature

$$
d s^{2}=e^{2 A_{s}(z)}\left(-f(z) d t^{2}+\frac{1}{f(z)} d z^{2}+d x_{i} d x^{i}\right)
$$

the equation of motion for $\chi(z)$ can be derived as

$$
\chi^{\prime \prime}+\left(3 A_{s}^{\prime}-\Phi^{\prime}+\frac{f^{\prime}}{f}\right) \chi^{\prime}+3 \frac{e^{2 A_{s}}}{f} \chi=0 .
$$

As in the original soft-wall model, we take the dilaton field of the simple quadratic form

$$
\Phi(z)=\mu^{2} z^{2},
$$

and the background metric as the AdS-Schwarzchild black hole solution

$$
\begin{gathered}
A_{s}(z)=-\log (z), \\
f(z)=1-\frac{z^{4}}{z_{h}^{4}} .
\end{gathered}
$$

Here $z_{h}$ is the horizon of the black hole defined at $f\left(z_{h}\right)=0$ and related to the temperature $T$ of the system by the Hawking temperature formula

$$
T=\left|\frac{f^{\prime}\left(z_{h}\right)}{4 \pi}\right|=\frac{1}{\pi z_{h}} .
$$

At zero temperature, we have $f(z) \equiv 1$, eq. (2.18) could be analytically solved as

$$
\chi(z)=c_{2} G_{1,2}^{2,0}\left(-\left.\mu^{2} z^{2}\right|_{\frac{1}{2}, \frac{3}{2}} ^{1}\right)+c_{1} e^{\frac{\mu^{2} z^{2}}{2}} \mu^{3} z^{3}\left(I_{0}\left(\frac{\mu^{2} z^{2}}{2}\right)+I_{1}\left(\frac{\mu^{2} z^{2}}{2}\right)\right) .
$$

with $I_{n}(z)$ the modified Bessel function of the first kind and $G_{p q}^{m n}\left(z \mid \begin{array}{c}a_{1}, \ldots, a_{p} \\ b_{1}, \ldots, b_{q}\end{array}\right)$ the MeijerG function. ${ }^{1}$ The $z \rightarrow \infty$ asymptotic behavior of eq. (2.23) is either exponentially blowing up or approaching a constant, which can be seen by taking two limits: $\chi^{\prime \prime} \ll \chi$, then $-2 \mu^{2} z \chi^{\prime}+3 \chi / z^{2}=0$ and $\chi_{1} \rightarrow e^{-3 /\left(4 \mu^{2} z^{2}\right)} \rightarrow 1 ; \chi^{\prime} \gg \chi$, then $\chi^{\prime \prime}-2 \mu^{2} z \chi^{\prime}=0$ and

\footnotetext{
${ }^{1}$ For details, please refer to Mathematica 10.
} 
$\chi_{2} \rightarrow e^{\mu^{2} z^{2}} /(\mu z)$. The exponentially growing branch is unacceptable, since it breaks the Regge behavior of axial vector in soft-wall model. Then because both the two independent solutions in eq. (2.23) blow up in the IR region, we will require $c_{1} \propto c_{2}$ to cancel the exponential growing part. Finally, we can get the ultraviolet(UV) $z \rightarrow 0$ asymptotic behavior of the solution of eq. (2.23) as

$$
\begin{aligned}
\chi(z) & =c\left(\mu z+\mu^{3} z^{3}\left(-\frac{1}{2}+\gamma_{E}+\frac{\psi\left(-\frac{1}{2}\right)}{2}+\log (\mu z)\right)\right)+O\left(z^{4}\right) \\
& =c\left(\mu z+\mu^{3} z^{3}(0.095+\log (\mu z))\right)
\end{aligned}
$$

with $\gamma_{E}=0.577$ the Euler's constant and $\psi(z)$ the digamma function with $\psi\left(-\frac{1}{2}\right)=0.036$. Comparing eq. (2.6) and eq. (2.24), one gets $c=m_{q} \zeta / \mu$ and

$$
\frac{\sigma(T=0)}{m_{q} \mu^{2} \zeta^{2}}=0.095
$$

From this result, we can see that the chiral condensate in the original soft-wall model is induced by the quark mass, and in the chiral limit $m_{q} \rightarrow 0$ it tends to vanish. In this sense, the original soft-wall model can not describe the spontaneous chiral symmetry breaking of QCD vacuum. The symmetry breaking is actually an explicit one.

At finite temperature, eq. (2.18) can not be solved analytically. By requiring $\chi(z)$ taking the asymptotic behavior as eq. (2.5) at UV and all the quantities being regular at horizon $z_{h}$, the authors of ref. [77] get the numerical result as shown in the dashed line in figure 2(a) in case $m_{q}=\mu$. In the plot, we scaled all the quantities with dimension to dimensionless quantities using the only mass scale $\mu$ in the model. In figure 2(a), we also show the $m_{q}$ dependence of $\sigma(T)$ explicitly. From figure 2(b) we see that the $\sigma / m_{q}$ results do not depend on $m_{q}$, which shows that $\sigma(T)=m_{q} g(T)$ with $g(T)$ being independent of $m_{q}$. One can also find that the near $T=0$ value of $\sigma /\left(m_{q} \zeta^{2} \mu^{2}\right)$ is just the same value 0.095 as extracted analytically, which can be seen as a consistent check of the numerical method we used(We leave this part in appendix A).

In fact, it is also easy to show this conclusion analytically: since eq. (2.18) is linear, the solution would be of the general form $\chi(z)_{T}=c_{1}(T) g_{1}(z)+c_{2}(T) g_{2}(z)$. In general, since $f\left(z_{h}\right)=0$ appears in the denominator of eq. (2.18), one might expect that at least one of $g_{1}(z)$ and $g_{2}(z)$ is singular at $z=z_{h}{ }^{2}$ If both $g_{1}(z)$ and $g_{2}(z)$ are singular, then one could expect that by linear combining the two functions the singularity at $z_{h}$ should be canceled and one gets one singular and one regular new function (if not, both the two functions could not be considered as physical one). Without loss of generality, we can assume that $g_{1}(z)$ is the singular one and $g_{2}(z)$ is regular at $z_{h}$. Then, we could expect that the solution of $\chi(z)=c_{2}(T) g_{2}(z)$, in which case $\sigma(T) \propto c_{2}(T) \propto m_{q}$. Thus, we see that non-linear terms in the scalar potential might be necessary to realize the correct

\footnotetext{
${ }^{2}$ Probably, there might be some extreme cases when both $g_{1}(z)$ and $g_{2}(z)$ are non-singular. In these cases, $\sigma$ and $m_{q}$ would be independent on each other, since we can not find a natural boundary condition to get rid of one of the two independent solutions. Thus, we did not consider this case.
} 


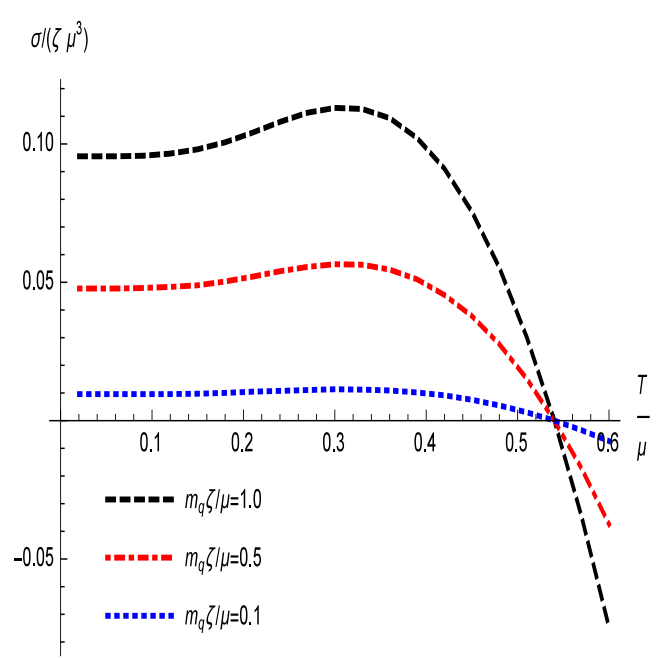

(a)

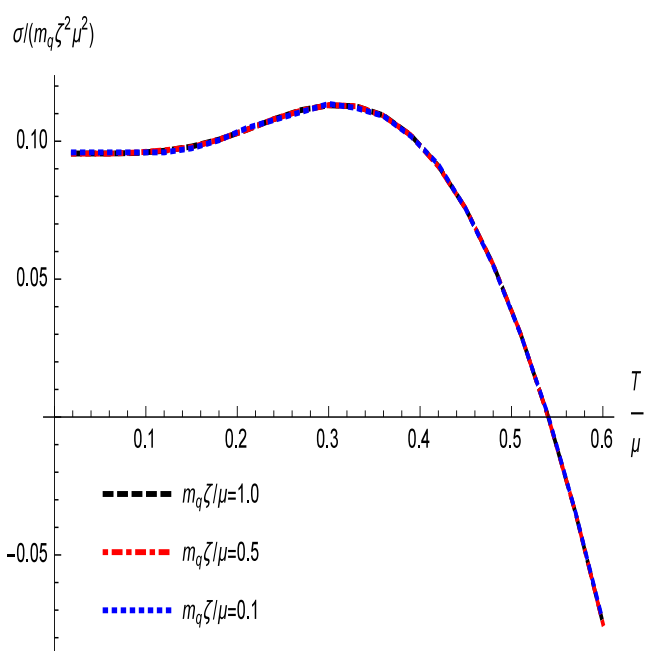

(b)

Figure 2. $\sigma(T)$ as a function of temperature $T$ in quadratic dilaton background eq. (2.19). Panel (a) gives the results of $\sigma /\left(\zeta \mu^{3}\right)$. The blue dotted, red dotdashed,and black dashed lines represent $m_{q} \zeta / \mu=0.1,0.5,1$ respectively. Panel (b) gives the $m_{q}$ rescaled dimensionless results $\sigma /\left(m_{q} \zeta^{2} \mu^{2}\right)$ of the three lines. All the three lines overlap and become the same curve, which shows that $\sigma / m_{q}$ does not depend on $m_{q}$.

behavior of chiral condensate (though it might be insufficient to only consider the nonlinear potential, since to induce the instability one might need to make the effective mass term violate the Breitenlohner-Freedman bound [81-83]).

In summary, the chiral condensate in the original model is induced by the quark mass, which is unreasonable from real QCD point of view. In addition, from the analysis, we could see that to add a non-linear term in the scalar potential might be necessary to get the correct spontaneous symmetry breaking behavior. Therefore, in the next section we will add non-linear terms in the potential of scalar field $X$ and try to solve this problem.

\section{Quartic potential effects}

In the previous section, we have seen that the model in section 2.2 can not give the correct chiral symmetry breaking mechanism at both zero and finite temperature. In order to modify the linear quark mass dependence behavior of chiral condensate $\sigma(T)$, it is necessary to add non-linear term in the potential of scalar field $X$, which has also been pointed out in [10] and has been studied in [19-25]. Here, we assume that the potential of scalar field $X$ takes a general form $V_{X}(|X|)$ in terms of $|X|$ and the action becomes

$$
S=-\int d^{5} x \sqrt{-g} e^{-\Phi} \operatorname{Tr}\left(D_{m} X^{+} D^{m} X+V_{X}(|X|)\right) .
$$

Inserting the expectation value of $X$, we get the the effective description in terms of $\chi$ of the following form

$$
S_{\chi}=-\int d^{5} x \sqrt{-g} e^{-\Phi}\left(\frac{1}{2} g^{z z} \chi^{\prime 2}+V(\chi)\right),
$$




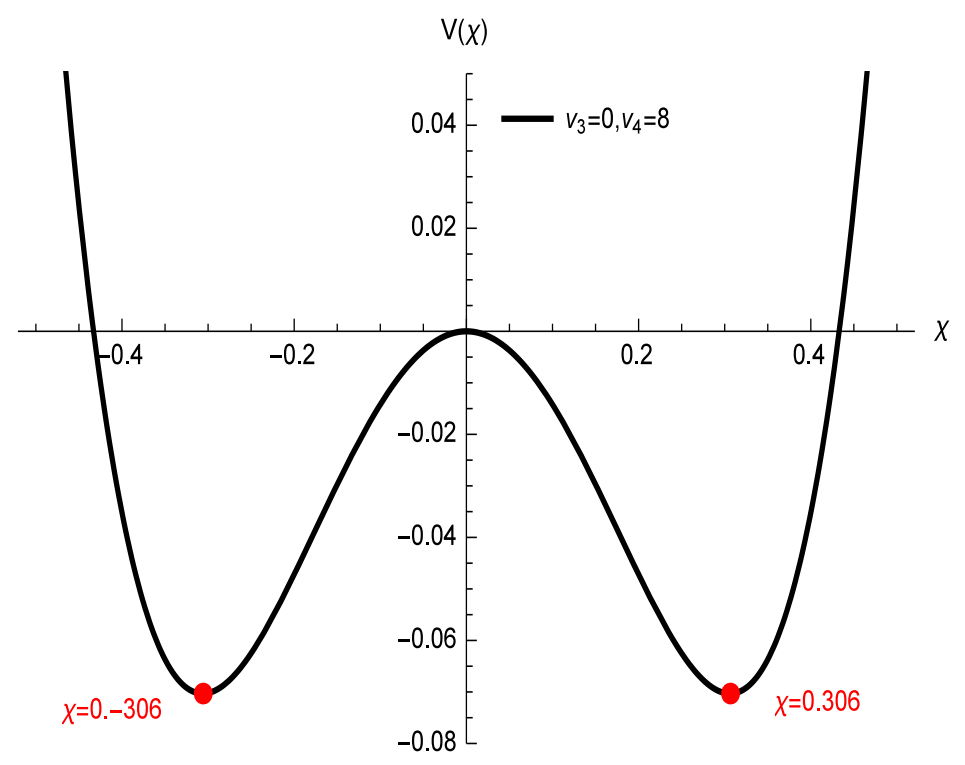

Figure 3. $V(\chi)$ as a function of $\chi$ with $v_{3}=0, v_{4}=8$. The two non-trivial solutions $\chi= \pm 0.306$ of $\partial_{\chi} V(\chi)=0$ are labeled.

where $V(\chi) \equiv \operatorname{Tr}\left(V_{X}(|X|)\right)$. The leading term of $V(\chi)$ comes from the mass term and it is fixed to be $-\frac{3}{2} \chi^{2}$ from AdS/CFT dictionary. To keep $\chi \leftrightarrow-\chi$ symmetry, the next power term is the quartic term $v_{4} \chi^{4}$. If one considers the $N_{f}=3$ case, there would be $\chi^{3}$ term coming from the t'Hooft determinant term $\operatorname{Re}[\operatorname{det}(X)]$. For later convenience, here we will consider

$$
V(\chi)=-\frac{3}{2} \chi^{2}+v_{3} \chi^{3}+v_{4} \chi^{4}
$$

In this section, we will only consider the $N_{f}=2$ case and only study the corrections from the quartic term when $v_{3}=0, v_{4} \neq 0$. Since in [19-22], $v_{4} \simeq 10$ gives a better prediction of meson spectral, in our work we will choose the parameter $v_{4}$ in this region. In figure 3 , we plot the potential of $v_{4}=8$, and we see that as in spontaneously broken of $\phi^{4}$ theory, $\partial_{\chi} V(\chi)=0$ has three solutions: $\chi=0$ and $\chi= \pm \sqrt{\frac{3}{4 v_{4}}} \simeq \pm 0.306$. We would see later that this structure plays an important role to realize the spontaneous symmetry breaking in $5 \mathrm{D}$.

The equation of motion for $\chi$ with respect to the action eq. (3.2) can be derived as

$$
\chi^{\prime \prime}+\left(3 A_{s}^{\prime}-\Phi^{\prime}+\frac{f^{\prime}}{f}\right) \chi^{\prime}-\frac{e^{2 A_{s}}}{f} \partial_{\chi} V(\chi)=0 .
$$

and we checked that the leading UV expansion of this equation is still of the form $m_{q} \zeta z+\frac{\sigma}{\zeta} z^{3}[80] .^{3}$

For later convenience in comparing the stability of different solutions, we would like to derive the free energy of the solutions here. One can use the equivalence of the partition

\footnotetext{
${ }^{3}$ Here and later, we only list the UV expansions with care and omit other possible expansion terms of orders in between $z$ and $z^{3}$, e.g. $z^{2}$ and $z^{3} \log (z)$. The coefficients of these terms are functions of $m_{q}$, and can be calculated order by order from eq. (3.4).
} 
function conjecture $Z_{Q C D}=Z_{\text {gravity }} \simeq e^{-S_{E}}$. Using the thermodynamical equality $Z=$ $e^{-\beta F}(\beta \equiv 1 / T)$, we have the relation between free energy $F$ and the on-shell Euclidean action of gravity $F=S_{E}$. Inserting eq. (3.4) into eq. (3.2), and notice the negative sign while doing the wick rotation, we have

$$
\begin{aligned}
\mathcal{F} \equiv \frac{F}{V_{3}} & =\int_{0}^{z_{h}} d z \sqrt{-g} e^{-\Phi}\left(\frac{1}{2} g^{z z} \chi^{\prime 2}+V(\chi)\right) \\
& =\int_{0}^{z_{h}} d z \sqrt{-g} e^{-\Phi}\left(\frac{1}{2} v_{3} \chi^{3}+v_{4} \chi^{4}\right)+\left.\frac{1}{2}\left(\chi e^{3 A_{s}-\Phi} f \chi^{\prime}\right)\right|_{\epsilon} ^{z_{h}} \\
& =-\int_{0}^{z_{h}} d z e^{5 A_{s}-\Phi}\left(\frac{1}{2} v_{3} \chi^{3}+v_{4} \chi^{4}\right)-\left.\frac{1}{2}\left(\chi e^{3 A_{s}-\Phi} f \chi^{\prime}\right)\right|_{\epsilon}
\end{aligned}
$$

where we have introduced the free energy density $\mathcal{F}$ to get rid of the infinite spatial volume $V_{3}$ in the integral of $S_{E}$.

When $m_{q}=0$, from the UV asymptotic behavior of $\chi$, it is easy to see that the last equation is regular at $\epsilon=0$ and the second term vanishes. In this case, $\chi \equiv 0$ is always a solution of eq. (3.4), since it satisfies $m_{q}=0$ and is regular everywhere. This solution stands for the chiral symmetry restored phase, for $\sigma=0$. When $N_{f}=2, v_{3}=0$, if $v_{4}>0$, we could easily see that $\mathcal{F}<0$ if $\chi \neq 0$, which means in chiral limit if there are non-trivial solutions of $\chi$, it is always thermodynamically more favored than the symmetry restored solution $\chi \equiv 0$. When $m_{q} \neq 0, \mathcal{F}$ is divergent near $\epsilon=0$, and in principle one has to add counter term to cancel the divergence. Since there could be scheme dependence when introducing the counter term, the exact value of chiral condensate would also be scheme dependent. Fortunately, focusing on the qualitative behavior of chiral phase transition, later we do not really need to deal with this case, so here we do not try to work out the explicit form of the counter terms. After all this preparation, in the following subsections, we will try to analyze the quark mass and temperature dependent behavior of $\sigma(T)$ under the quatric corrections in the potential.

\subsection{Chiral condensate in positive quadratic dilaton background}

Firstly, we work in the positive dilaton background as in the last section. We take $v_{4}=8$ and solve eq. (3.4) with the boundary condition $\chi(z \rightarrow 0) \rightarrow m_{q} z, \chi\left(z \rightarrow z_{h}\right)<\infty$. We take $m_{q} \zeta / \mu=0.003,0.015,0.03,0.1$ and show the results in figure 4. Since there is only one parameter with mass dimension, we plot the dimensionless combination $\sigma /\left(\zeta \mu^{3}\right)$ and $\sigma /\left(m_{q} \zeta^{2} \mu^{2}\right)$ in terms of $T / \mu$. It is very clear to see that those plots will not depend on $\mu$.

From panel (a), we can see chiral condensate at low temperature decrease with the decreasing of quark mass. From the plots of $\sigma /\left(m_{q} \zeta^{2} \mu^{2}\right)$ in panel (b), we could see that $\sigma$ is no longer simply proportional to $m_{q}$ due to the non-linear potential. In the large $m_{q}$ region, the non-linear potential would affect the result of $\sigma$ significantly. It increases the value of $\sigma /\left(m_{q} \zeta^{2} \mu^{2}\right)$ from 0.095 to around 0.2 at $T \simeq 0$ region. However, when $m_{q}$ approaching the chiral limit, it can be seen that $\sigma /\left(m_{q} \mu^{2} \zeta^{2}\right)$ will approach a limit, which is just the results without the quartic potential term. Therefore, we see that in the small $m_{q}$ region, $\sigma \propto m_{q}$ is approximately true, which again shows that no chiral condensate in chiral limit and the symmetry breaking is still an explicit one induced by quark mass. 


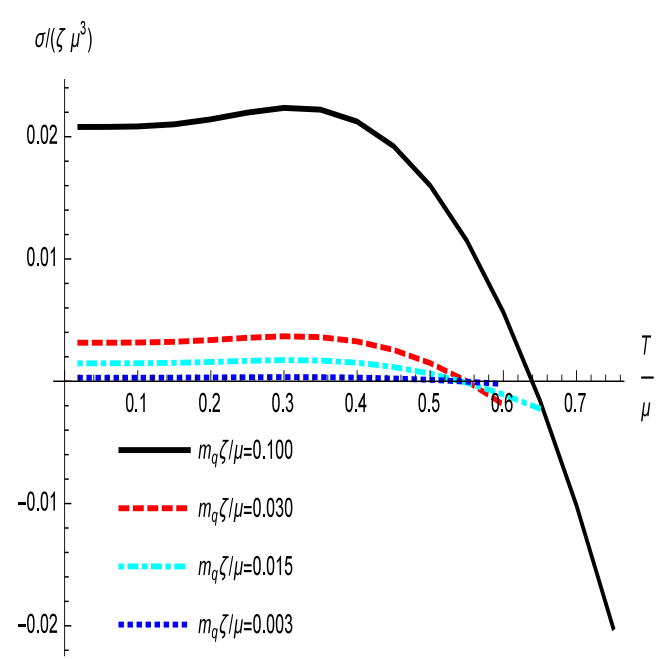

(a)

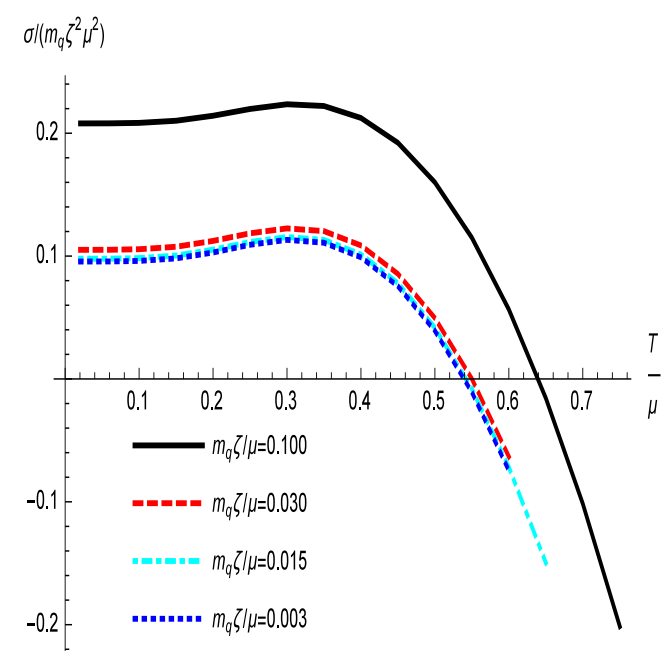

(b)

Figure 4. $\sigma(T)$ as a function of temperature $T$ in quadratic dilaton background eq. (2.19) with $v_{3}=0, v_{4}=8$. Panel (a) gives the results of $\sigma /\left(\zeta \mu^{3}\right)$. The blue dotted, cyan dotdashed, red dashed and black solid lines represent $m_{q} \zeta / \mu=0.003,0.015,0.03,0.1$ respectively. Panel (b) gives the $m_{q}$ rescaled dimensionless results $\sigma /\left(m_{q} \zeta^{2} \mu^{3}\right)$ of the three lines. When $m_{q} \rightarrow 0$, the results of $\sigma /\left(m_{q} \zeta^{2} \mu^{3}\right)$ tend to be independent of $m_{q}$ and approach the lines in figure 2(b).

From the above discussion, unlike the 4D field theory, it is insufficient to get spontaneously symmetry breaking with only the $\phi^{4}$ like potential. Modification on the dilaton field or the metric background is necessary.

\subsection{Chiral condensate in negative dilaton model}

The positive dilaton model does not give the correct spontaneous symmetry breaking behavior in chiral limit. In this section, as a test, we will work in the negative dilaton background

$$
\Phi(z)=-\mu^{2} z^{2},
$$

though as pointed out in $[10,24,84]$ it might cause an un-physical massless scalar meson state.

Firstly, we take $v_{4}=8$ and consider the chiral limit case. Taking $m_{q}=0$ in the UV expansion $\chi(z)=m_{q} \zeta z+\sigma / \zeta z^{3}+\ldots$ and requiring $\left|\chi\left(z_{h}\right)\right|<\infty$ at horizon, we could solve $\sigma(T)$ from eq. (3.4) using the method described in appendix A. The results are shown in figure 5 , in which we have scaled all the quantities with dimension into dimensionless combination using $\mu$. From figure 5(a), we could see that below $T=0.198 \mu$ there are two non-trivial $\sigma \neq 0$ solutions labeled by red and blue solid lines together with the trivial $\chi \equiv 0$ solution labeled by cyan solid line. In fact, the red and blue solutions are just the $\sigma \leftrightarrow-\sigma$ reflection of each other, which is the manifestation of the $\chi \leftrightarrow-\chi Z_{2}$ symmetry of the scalar potential eq. (3.3) when $v_{3}=0$. Thermodynamically, the non-trivial solutions are more stable than the trivial $\sigma=0$ solution when $v_{3}=0$, as has been explained at the beginning of this section. Furthermore, the non-trivial solutions of $\sigma$ approach a finite 


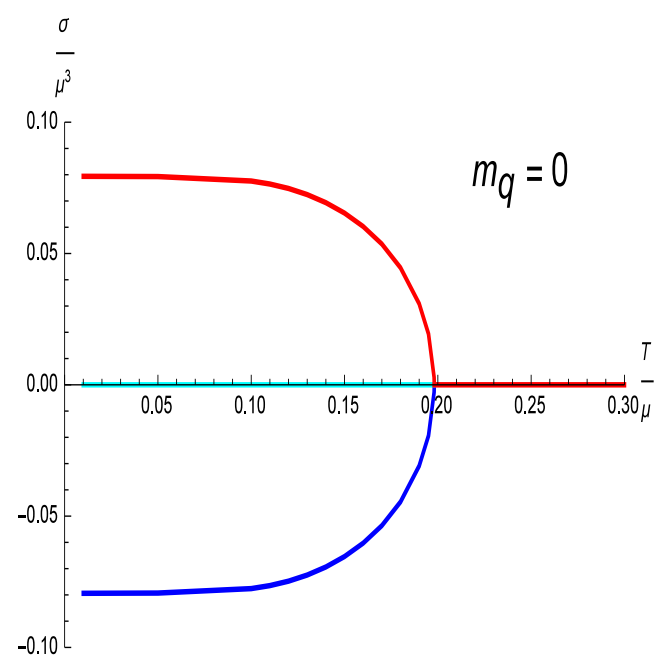

(a)

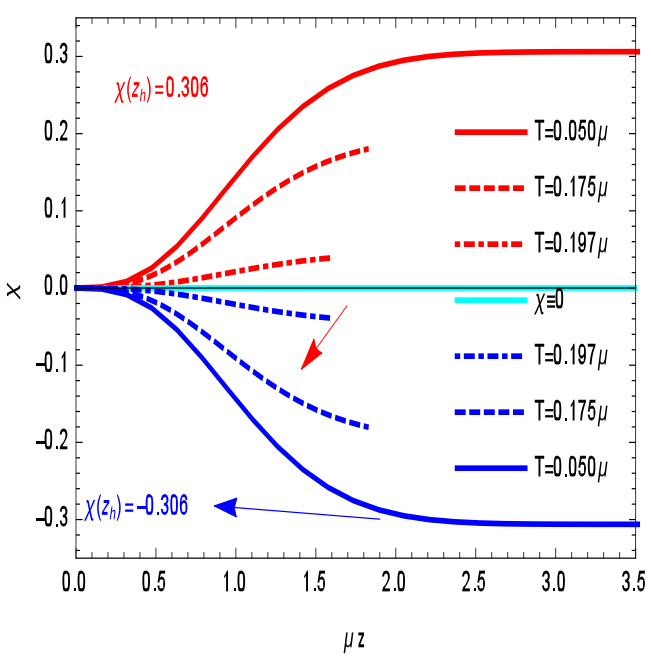

(b)

Figure 5. Panel (a) shows $\mu^{3}$ scaled dimensionless chiral condensate $\sigma(T) / \mu^{3}$ as a function of $\mu$ scaled temperature $T / \mu$ in negative quadratic dilaton background eq. (3.6) when $v_{3}=0, v_{4}=8$ and $m_{q}=0$. Panel (b) shows the corresponding $\chi$ solutions when $T=0.050,0.175,0.197 \mu$. We only plot the part outside the black hole $0<z<z_{h}=1 /(\pi T)$ in each temperature and cut the long constant tail in the region $3.5<\mu z<20 / \pi$ when $T=0.05 \mu$ for compactness of the plots.

constant value $\sigma_{0} \equiv \sigma(T=0) \simeq 0.08 \mu^{3}=(0.43 \mu)^{3}$ at low temperature, representing the spontaneous chiral symmetry breaking in the vacuum. Then, when $T$ increasing, $\sigma(T)$ decreases slowly from $0.08 \mu^{3}$ to around $0.07 \mu^{3}$ in the temperature region $0<T<0.140 \mu$. Nextly, when the temperature continues to increase from $0.140 \mu$ to $0.198 \mu, \sigma(T)$ decreases rapidly from $0.07 \mu$ to zero. Above $T=0.198 \mu$, we can not find non-trivial solutions, which shows the restoration of chiral symmetry at high temperature. From this picture, we have realized the spontaneous chiral symmetry breaking in the vacuum $T=0$ and its restoration above the critical temperature $T_{C}^{\text {negative }}=0.198 \mu$. The phase transition is a second order one in chiral limit, consistent with the 'Columbia sketch plot' in two flavor chiral limit. We also note that if we take $\mu=0.75 \mathrm{GeV}$, then vacuum value of chiral condensate and the critical temperature would be $\sigma_{0} \simeq(323 \mathrm{MeV})^{3}$ and $T_{C}^{\text {negative }} \simeq 150 \mathrm{MeV}$, which is comparable with the lattice results [73, 74].

In order to show the effects of the two non-trivial vacua in scalar potential eq. (3.3) and figure 3, we also plot the corresponding solutions of $\chi(z)$ at temperature $T=$ $0.05,0.175,0.197 \mu$ in figure $5(\mathrm{~b})$. From this figure, we could see that at low temperature $T=0.05 \mu, \chi(z)$ would increase from 0 to a finite constant value near the horizon. Actually, we find that even when $T$ is around $0.1 \mu$, the configuration of $\chi(z)$ does not change too much. We extract the near horizon value and find that it equals to 0.306 , which is just the too non-trivial vacuum labeled in figure 3 . Then when temperature increases, we see that the solution of $\chi(z)$ falls towards the $x$ axis, and when $T=0.197 \mu \chi(z)$ become very close to $\chi=0$. It is easy to imagine that when $T=T_{C}^{\text {negative }}$, the solution of $\chi(z)$ become exactly $\chi(z)=0$ at range of $0<\mu z<1 /(\pi T)$. Therefore, we see that the near $T=0$ solutions of $\chi(z)$ is just the interpolation between the trivial vacuum $\chi=0$ and 


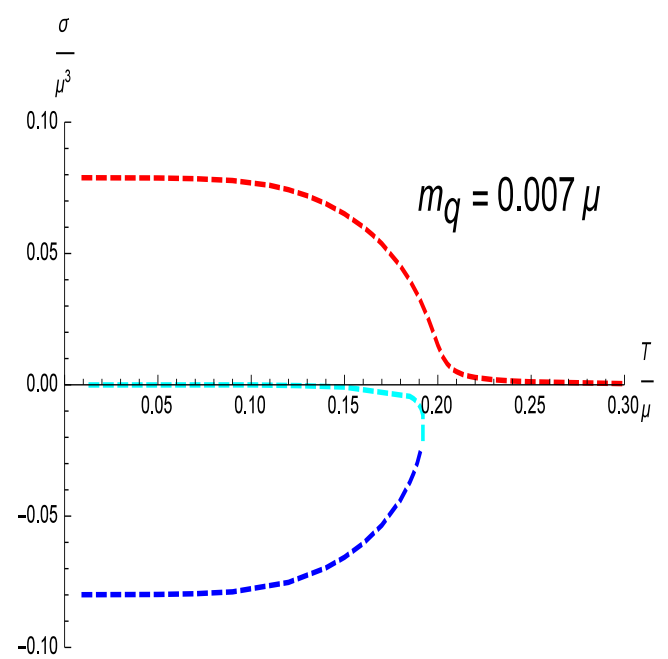

(a)

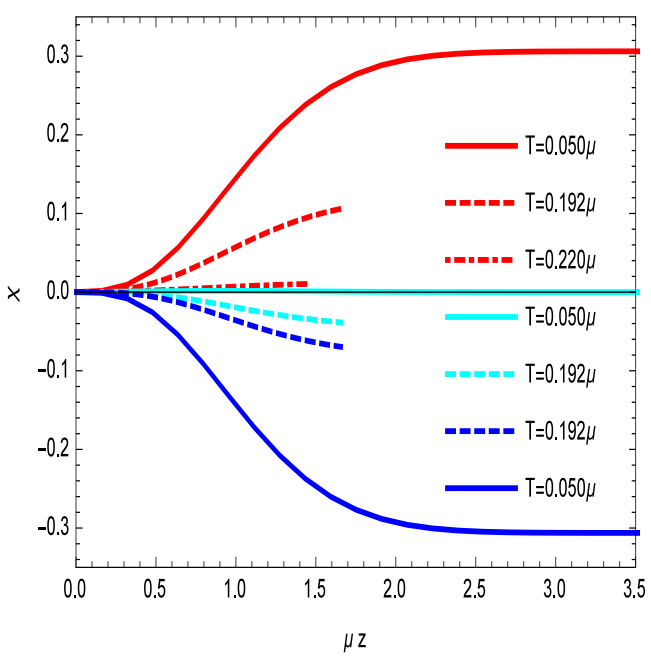

(b)

Figure 6. Panel (a) shows $\mu^{3}$ scaled dimensionless chiral condensate $\sigma(T) / \mu^{3}$ as a function of $\mu$ scaled temperature $T / \mu$ in negative quadratic dilaton background eq. (3.6) when $v_{3}=0, v_{4}=8$ and $m_{q}=0.007 \mu$. Panel (b) shows the corresponding $\chi$ solutions when $T=0.050,0.192,0.220 \mu$. We only plot the part outside the black hole $0<z<z_{h}=1 /(\pi T)$ in each temperature and cut the long constant tail in the region $3.5<\mu z<20 / \pi$ when $T=0.05 \mu$ for compactness of the plots.

the two non-trivial vacuum $\chi= \pm 0.306$, which in some sense shows the necessity of the positive quartic term in the scalar potential.

Now that the chiral phase transition in chiral limit is described quite well in this model, we would go further and try to investigate its finite quark mass behavior. We take $m_{q}=0.007 \mu$ as an example and plot the results in figure 6 . We would emphasize that the qualitative results for any finite quark mass are the same, though for simplicity we only show only one of them here. From figure 6(a), we see that the positive quark mass $m_{q}=0.007 \mu$ would break the $\sigma \leftrightarrow-\sigma$ symmetry of the solution. As a result, the trivial $\chi \equiv 0$ would not be a solution any more, since it does not satisfy the boundary condition $\chi(z)=m_{q} \zeta z+\ldots$ at UV. Comparing to the chiral limit result figure 5 , we could see that near $T=0$ the results do not change too much and the vacuum value of $\sigma$ is still around $0.08 \mu^{3}$. However, when $T>0.15 \mu$, the low $T$ cyan part and the high $T$ red part of the trivial solution $\chi \equiv 0$ in figure 5 (a) would be seperated when $m_{q}>0$ in figure 6 (a). The cyan part is dragged down from the $x$ axis and bent towards the negative $\sigma$ branch, while the red part would be dragged up and bent towards the positive $\sigma$ branch. The blue non-trivial solution and the quark mass induced cyan part would join together at around $T=0.195 \mu$, while the quark mass induced red part would join the red non-trivial solution and become a continuous line.

In figure 6(b), we also plot the solutions of $\chi(z)$ at different temperature $T=$ $0.05,0.192,0.22 \mu$. From the figure, we could also see the same qualitative picture from the evolution of $\chi$. At low temperature like $T=0.05$, the three solutions do not change too much, and the cyan line almost equals to 0 . Then the increasing of temperature would drag the cyan lines down towards the blue negative $\sigma$ solutions, and it could be imag- 


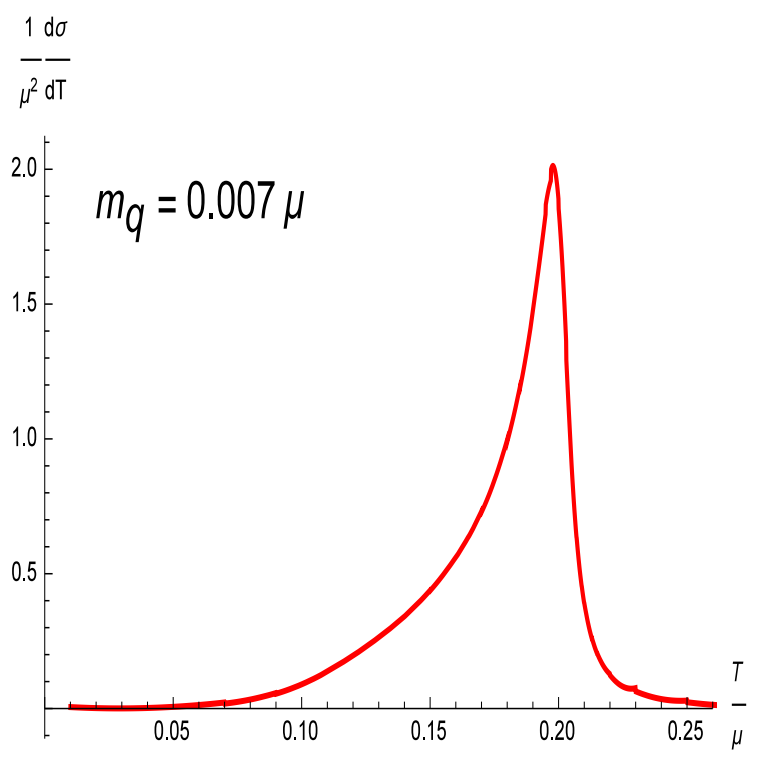

Figure 7. $\mu^{2}$ scaled $\frac{d \sigma(T)}{d T}$ as a function of $T$ in negative quadratic dilaton background eq. (3.6) when $v_{3}=0, v_{4}=8$ and $m_{q}=0.007 \mu$.

ined that at temperature above $T=0.192 \mu$ they would merge to be the same solution. Meanwhile, the red lines are dragged down towards the $x$ axis continuously.

From the red dashed line in figure 6(a), we see that at finite quark mass the phase transition would become a crossover one, since the finite quark mass would destroy the $\sigma \leftrightarrow-\sigma$ symmetry in the 5D theory. The crossover transition is not a real phase transition, and we would define the transition point as the location of largest $\frac{d \sigma}{d T}$. We plot the results of $\frac{d \sigma}{d T}$ in figure 7 , and there we can see that since the quark mass we take are quite small, the transition temperature is almost the same as that in chiral limit, i.e. $T_{C}^{\text {negative, } m} \simeq 0.198 \mu$.

Thus, the negative dilaton plus the quartic scalar potential do give a very good realization of spontaneous chiral symmetry breaking in the vacuum and its restoration at high temperature. The transition is of second order type in chiral limit and it would turn to be a crossover one in any finite quark mass. However, as pointed out in [10, 24, 84], the large $z$ negative dilaton would cause an un-physical massless scalar meson state. Therefore, we will modify the large $z$ behavior of dilaton field and try to cure this problem in the next section.

\section{Interpolated dilaton: two different scales}

From the above discussion, we find that the negative dilaton gives a prediction on chiral phase transition agreeing perfectly well with the Columbia sketch plot whereas the positive dilaton background can not generate correct results in chiral limit. However, as pointed out in $[10,24,84]$, the negative dilaton background predicts an un-physical massless scalar meson state which is unacceptable. The results from spectra analysis and thermodynamical analysis seem in contradiction. To solve this issue, one has to note that the dominating energy scale of chiral symmetry breaking is around $1 \mathrm{GeV}$ and that of confinement is around 
$200 \sim 300 \mathrm{MeV}[85]$. In the Regge behavior analysis, the confinement is the dominating effect while in chiral symmetry breaking mechanism new scale should be introduced. Taking into account the latter, we expect that the negative dilaton dominates at small $z$ and at large $z$ positive dilaton would dominate. In between the two, we take the following simple interpolation as a test

$$
\Phi(z)=-\mu_{1}^{2} z^{2}+\left(\mu_{1}^{2}+\mu_{0}^{2}\right) z^{2} \tanh \left(\mu_{2}^{2} z^{2}\right),
$$

where $\mu_{0}$ would be fixed to around $0.43 \mathrm{GeV}$ as the extended soft-wall models [19-26] to produce the correct Regge slope in the highly excited states, and $\mu_{1}, \mu_{2}$ are two free parameters. At UV, $\Phi(z) \rightarrow-\mu_{1}^{2} z^{2}$, and at IR the above interpolation form goes to $\Phi(z) \rightarrow \mu_{0}^{2} z^{2}$, which is responsible for the linear confinement. In this section, we will focus on solving the structure of chiral condensate under the dilaton profile eq. (4.1) and the scalar potential eq. (3.3).

\section{1 $\quad \mathrm{SU}(2)$ case: $v_{3}=0, v_{4} \neq 0$}

Since we fix $\mu_{0}=0.43 \mathrm{GeV}$ for the Regge slope, there are 4 free parameters $\mu_{1}, \mu_{2}, v_{3}, v_{4}$ under the simple selection of dilaton profile eq. (4.1) and the scalar potential eq. (3.3). As we mentioned in section 3, the t'Hooft determinant term would only appear in SU(3) case, since $\operatorname{det}[X]$ is a $\chi^{2}$ term in $\mathrm{SU}(2)$ case. In this section, we would focus on $\mathrm{SU}(2)$ case first, so we will take $v_{3}=0$. Thus, the only free parameters left are $\mu_{1}, \mu_{2}$ and $v_{4}$. In principle, we could fix these parameters by comparing the predicted meson spectral to the experimental data. However, considering that to get a better fitting of the experimental data quantitatively, the metric as well as the $5 \mathrm{D} M_{5}^{2}$ could be modified as a more general $z$ dependent function like in $[23,69]$. Here we would like to focus on the qualitative results and leave the careful quantitative study to the future. Since qualitatively, the model we are considering could well describe the linear confinement behavior at zero temperature, we would like to investigate the qualitative behavior of the chiral dynamics in this model.

Firstly, we would study the $\mu_{1}, \mu_{2}$ dependence of the model results in chiral limit. We take $v_{4}=8$ as in the negative dilaton case and $m_{q}=0$. As an example, we fix $\mu_{2}=0.176 \mathrm{GeV}$, then we tune $\mu_{1}$ and try to solve $\sigma(T)$ from eq. (3.4). We plot the results in figure 8. From figure 8(a), the negative part of dilaton field would be shifted up when one decrease $\mu_{1}$, while the large $z$ quadratic tails stay in the same limit $\mu_{0}^{2} z^{2}$. From figure 8(b), we can see that as in the negative dilaton model, the chiral condensate only appears in low temperature region. The transition temperature where the non-trivial solution start disappearing would increase with the increasing of $\mu_{1}$, i.e. the increasing of the negative area in dilaton profile. When $\mu_{1}=0.75,0.83 \mathrm{GeV}$, chiral condensate would decrease monotonous from a finite value to zero, while for $\mu_{1}=0.606,0.65 \mathrm{GeV}$ chiral condensate would increase with temperature first and then decrease rapidly to zero.

Next, we fix $\mu_{1}=0.83 \mathrm{GeV}$, take $v_{4}=8, m_{q}=0$ and turn to study the $\mu_{2}$ effects. The results are shown in figure 9. Similarly, from the figure we see that when we increase $\mu_{2}$ the negative part of dilaton would be smaller and the transition temperature and chiral condensate would decrease. Moreover, when the negative part of dilaton profile becomes 


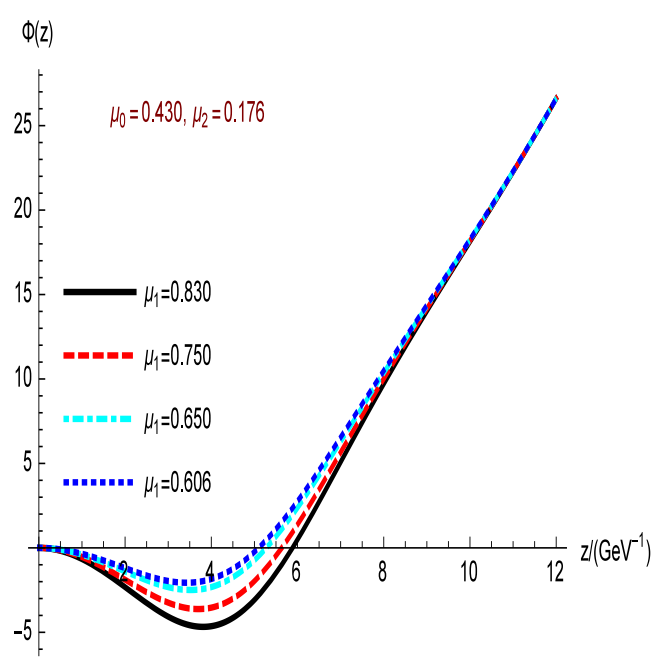

(a)

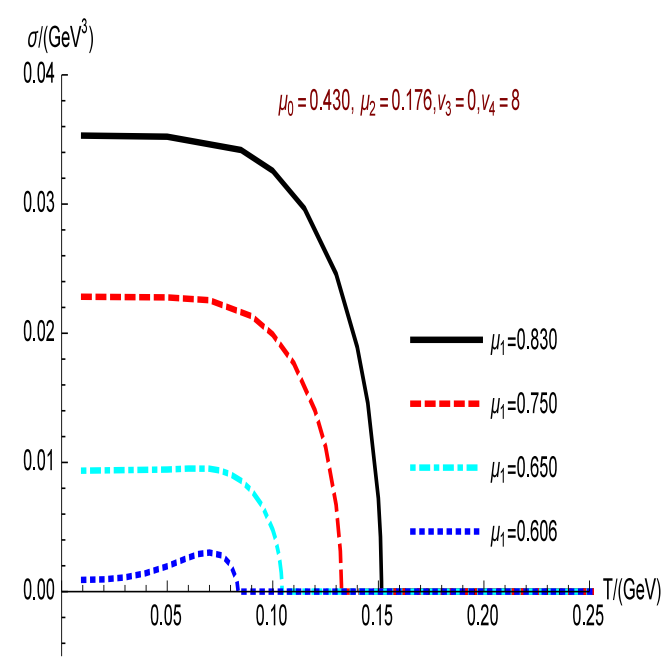

(b)

Figure 8. $\mu_{1}$ dependence of $\Phi(z)$ in eq. (4.1) and the corresponding results of $\sigma(T)$ as a function of temperature $T$ when $v_{3}=0, v_{4}=8$ and $\mu_{0}=0.43 \mathrm{GeV}, \mu_{2}=0.176 \mathrm{GeV}$. In panel (a), the black solid, red dashed, cyan dotdashed, and blue dotted lines give the plots of $\Phi(z)$ when $\mu_{1}=$ $0.83,0.75,0.65,0.606 \mathrm{GeV}$ respectively. Here we only plot the $\sigma>0$ part, and the $\sigma<0$ part can be easily got by reflecting the results along $T$ axis. In panel (b) $\sigma(T)$ in chiral limit are shown in lines with the same symbols correspondingly.

small, chiral condensate starts to increase at low temperature region and decrease fast to zero near the transition temperature.

From the above dependence behavior of $\mu_{1}, \mu_{2}$, we could see that a larger negative part of dilaton would favor a larger $\sigma$ in the vacuum and low temperature region. This is consistent with our previous study on the positive and negative quadratic models, in which no negative part of dilaton field is corresponding to zero chiral condensate and full negative part gives finite condensate in chiral limit. In addition, this also shows that it is the intermediate part of the dilaton dominating in chiral dynamics, since the large $z$ behavior of the model is universal for different parameter values.

Finally, we take $\mu_{1}=0.83 \mathrm{GeV}, \mu_{2}=0.176 \mathrm{GeV}$ and study the model dependence of $v_{4}$. The results are presented in figure 10. From figure 10(a), we can see that increasing $v_{4}$ would decrease the non-trivial vacuum $\chi_{0}= \pm \sqrt{\frac{3}{4 v_{4}}}$. Correspondingly, the chiral condensate would decrease, as can be seen from figure 10(b). However, unlike decreasing $\mu_{1}$ and increasing $\mu_{2}$, the transition temperature would stay the same when increase $v_{4}$.

Based on the above studies, we take $\mu_{1}=0.83 \mathrm{GeV}, \mu_{2}=0.176 \mathrm{GeV}, \mathrm{v}_{4}=8$ and turn to study the effects of quark mass. As before, we plot the results of chiral limit in figure 11 first. From figure 11(a), we can read that the vacuum value of chiral condensate $\sigma_{0}$ is around $0.035 \mathrm{GeV}^{3} \simeq(327 \mathrm{MeV})^{3}$ and the transition temperature $T_{C}^{\mathrm{SU}(2), 0}$ is around $151 \mathrm{MeV}$. Both of them are comparable with the lattice results. Furthermore, since in chiral limit the free energy formula eq. (3.5) is finite near the boundary $z=0$, we insert the $\chi(z)$ solutions into this formula and get the free energy results as shown in figure 11(b). 


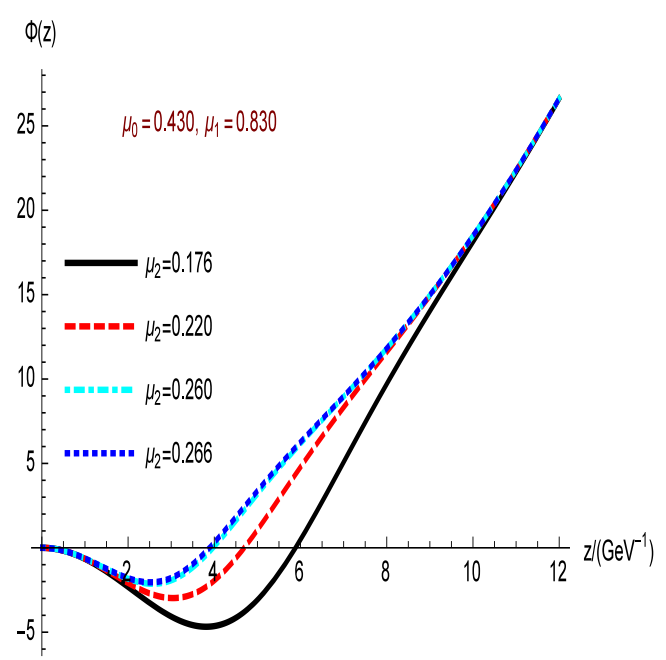

(a)

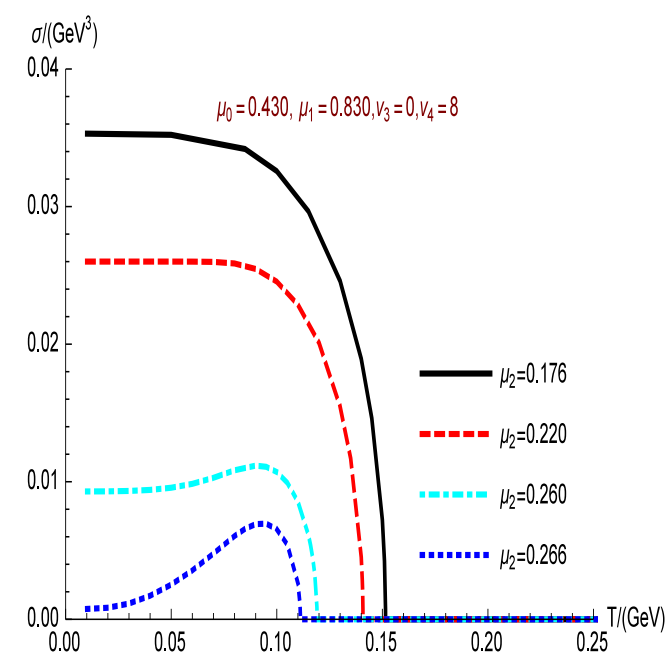

(b)

Figure 9. $\mu_{2}$ dependence of $\Phi(z)$ in eq. (4.1) and the corresponding results of $\sigma(T)$ as a function of temperature $T$ when $v_{3}=0, v_{4}=8$ and $\mu_{0}=0.43 \mathrm{GeV}, \mu_{1}=0.83 \mathrm{GeV}$. In panel (a), the black solid, red dashed, cyan dotdashed, and blue dotted lines give the plots of $\Phi(z)$ when $\mu_{2}=$ $0.176,0.22,0.26,0.266 \mathrm{GeV}$ respectively. In panel (b) $\sigma(T)$ in chiral limit are shown in lines with the same symbols correspondingly.

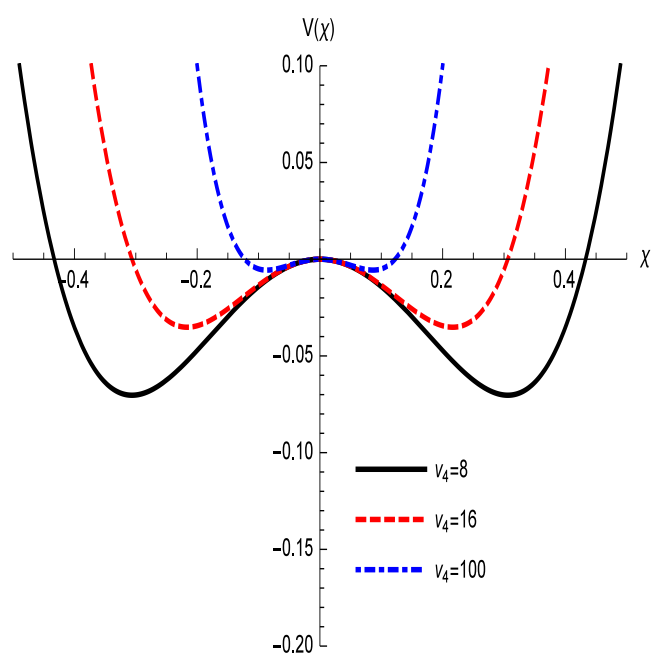

(a)

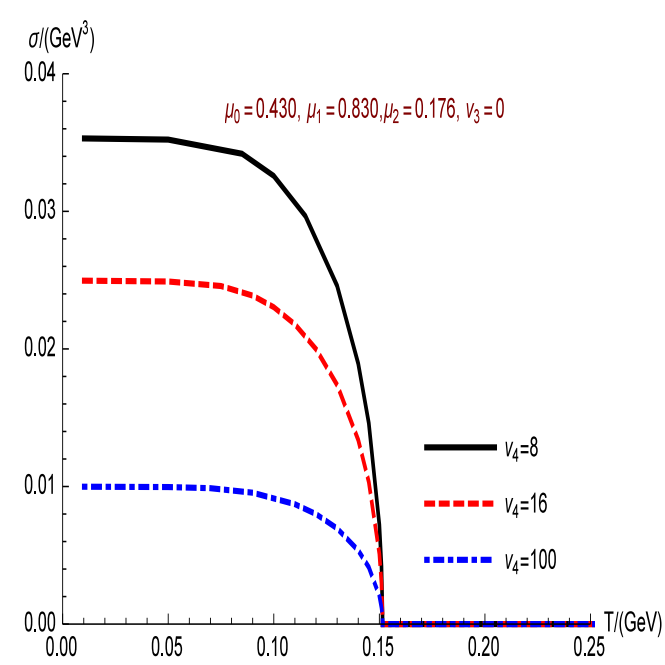

(b)

Figure 10. $v_{4}$ dependence of the scalar potential $V(\chi)$ in eq. (3.3) and the corresponding results of $\sigma(T)$ as a function of temperature $T$ when $v_{3}=0$ and $\mu_{0}=0.43 \mathrm{GeV}, \mu_{1}=0.83 \mathrm{GeV}, \mu_{2}=$ $0.176 \mathrm{GeV}$. In panel (a), the black solid, red dashed and blue dotdashed lines give the plots of $V(\chi)$ when $v_{4}=8,16,100$ respectively. In panel (b) $\sigma(T)$ in chiral limit are shown in lines with the same symbols correspondingly. 


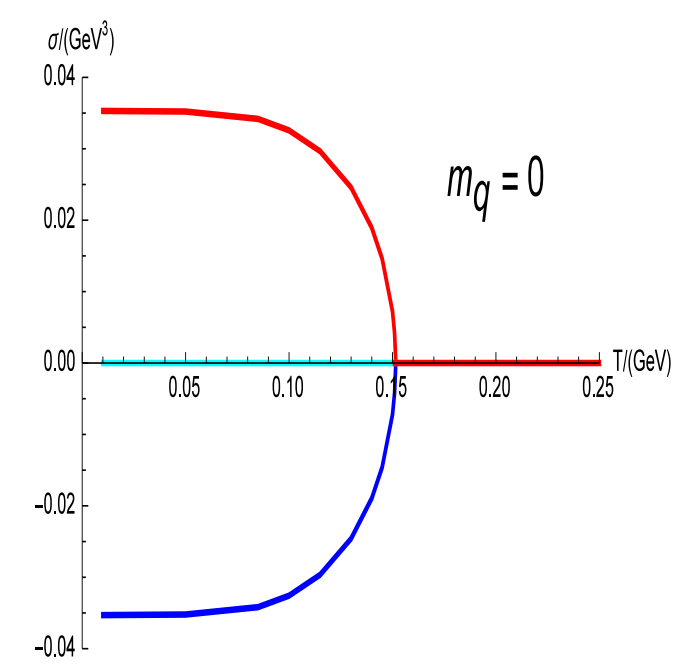

(a)

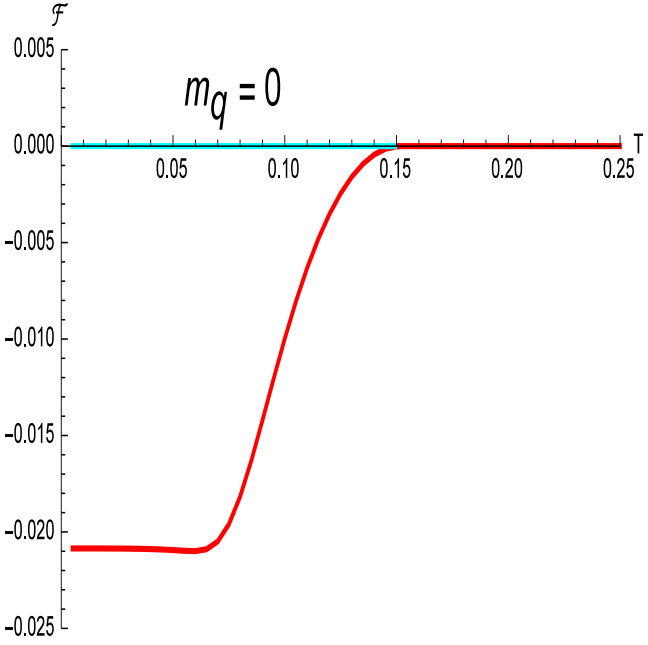

(b)

Figure 11. The results of chiral condensate $\sigma(T)$ and the corresponding free energy density $\mathcal{F}$ as a function of temperature $T$ in dilaton background eq. (4.1) when $v_{3}=0, v_{4}=8$ and $\mu_{0}=$ $0.43 \mathrm{GeV}, \mu_{1}=0.83 \mathrm{GeV}, \mu_{2}=0.176 \mathrm{GeV}$. Panel (a) shows the result of $\sigma(T)$ in chiral limit and panel (b) compares the free energy density $\mathcal{F}$ of different solutions.

From this figure, we could see that the free energy of the non-trivial solutions are always smaller than the trivial solutions. Besides, near the transition point, we find that the curve of non-trivial solutions is tangent to the trivial solution curve. This fact confirms that the phase transition in chiral limit is a second order one.

After turning on the mass source, we find that any finite quark mass would change the phase transition order. As an explicit example, we take $m_{q}=7 \mathrm{MeV}$ and show the results in figure 12. As can be seen from figure 12, the qualitative results are totally the same as in the negative dilaton model described in section 3.2. A positive quark mass would shift up the high temperature trivial solutions, join the positive branches of non-trivial solutions and merge to be one continuous line. The low temperature trivial solutions would be shift down. It would join the negative non-trivial solutions and merge to be a semi-circle appearing only at temperature below $147 \mathrm{MeV}$. Therefore, the physical line should be the continuous red line in figure 12. To extract the pseudo-transition temperature, we calculate $\frac{d \sigma}{d T}$ and read the transition temperature from the peak of figure 12(b), which gives $T_{C}^{\mathrm{SU}(2), m} \simeq 150 \mathrm{GeV}$, almost the same as the chiral limit.

As a short summary of this section, the interpolating dilaton model does give correct behavior of chiral phase transition. In chiral limit, it does give spontaneous chiral symmetry breaking in the vacuum and its restoration at high temperature. We also confirm that the transition order is of second order. At any finite quark mass the transition turns to be a crossover one. From the current study, the negative part in the intermediate scale is important for spontaneous chiral symmetry breaking and the positive quadratic part is important for linear confinement. Qualitatively, this is consistent with the studies in [85], though we do not know how to map the exact value of the energy scales. It should also 


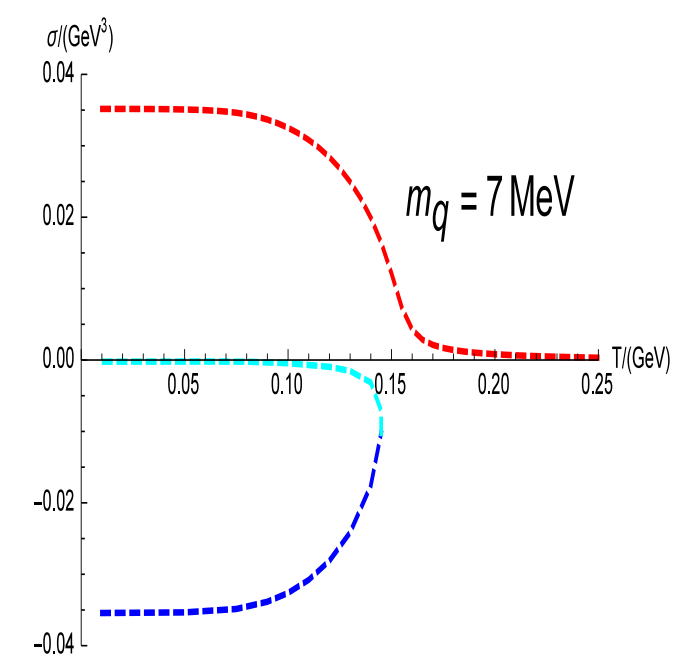

(a)

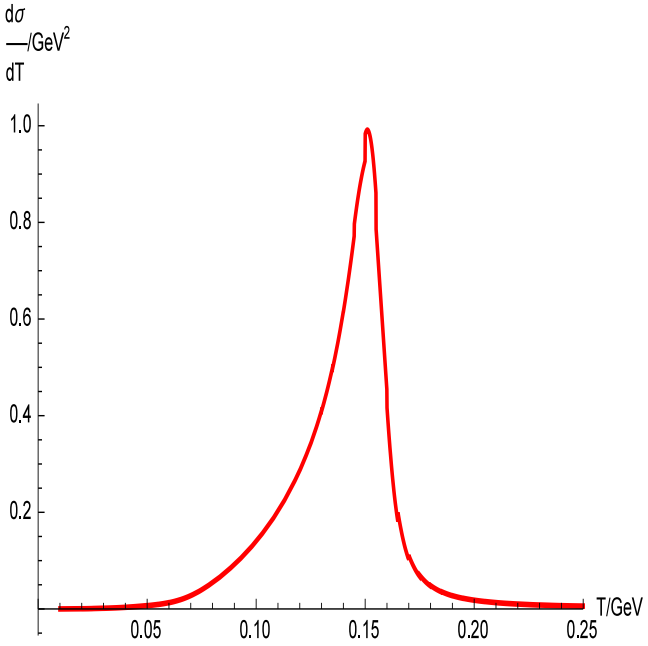

(b)

Figure 12. The results of chiral condensate $\sigma(T)$ and its temperature derivative $\frac{d \sigma(T)}{d T}$ as a function of temperature $T$ in dilaton background eq. (4.1) when $v_{3}=0, v_{4}=8$ and $\mu_{0}=0.43 \mathrm{GeV}, \mu_{1}=$ $0.83 \mathrm{GeV}, \mu_{2}=0.176 \mathrm{GeV}$. Panel (a) shows the results of $\sigma(T)$ when $m_{q} \simeq 7 \mathrm{MeV}$. Panel (b) gives the plot of $\frac{d \sigma(T)}{d T}$, the local maximum of which gives the pesudo transition temperature.

be noted that, here we choose a simple interpolating form of the dilaton field, which could be carefully tuned to accommodate the meson spectra also. In this work, we focus only on producing the correct qualitative behavior of chiral phase transition.

\section{$4.2 \mathrm{SU}(3)$ case: $v_{3}, v_{4} \neq 0$}

In the previous section, we have realized the top line of the phase diagram in mass plane, which is shown in figure 1. In this section, we would like to test this model in the $\mathrm{SU}(3)$ case, i.e. the diagonal line in figure 1.

In the $\mathrm{SU}(3)$ case, if we only consider the quartic term $X^{4}$, then it is easy to understand that the results would not be different from the $\mathrm{SU}(2)$ case. Fortunately, it is possible to introduce the t'Hooft determinant term $\operatorname{det}[X]$. If we consider a more general $N_{f}=2+1$ case, the vacuum expectation value of $X$ should be written as $X=\operatorname{diag}\left\{\chi_{l}, \chi_{l}, \chi_{s}\right\}$. Then the mass term and the quartic term would be proportional to $2 \chi_{l}^{2}+\chi_{s}^{2}$ and $2 \chi_{l}^{4}+\chi_{s}^{4}$ respectively. There is no coupling term in between $\chi_{l}$ and $\chi_{s}$. It is different for the determinant term, which reduces to $\chi_{l}^{2} \chi_{s}$ and cause the mixing of light flavor and heavy one. In principle, we can study the whole plane in figure 1 . However, it is much more complicated to solve the two coupled equations. So, we would leave this calculation to the future and focus on the $\mathrm{SU}(3)$ case only. When $m_{u}=m_{d}=m_{s}$, one can expect that $\chi_{l}=\chi_{s}$, then the above settings would reduce to the model described in previous section with finite $v_{3}$.

Since we are more interested in the qualitative result, instead of fixing $v_{3}, v_{4}$ from meson spectra, we would only take $v_{3}=-3, v_{4}=8$ as an example to show the qualitative behavior. The potential are shown in figure 13. In the figure, we find that the $\chi \leftrightarrow-\chi$ symmetry is broken explicitly by the cubic potential. The right vacuum is around $\chi^{(+)}=0.477$ and 


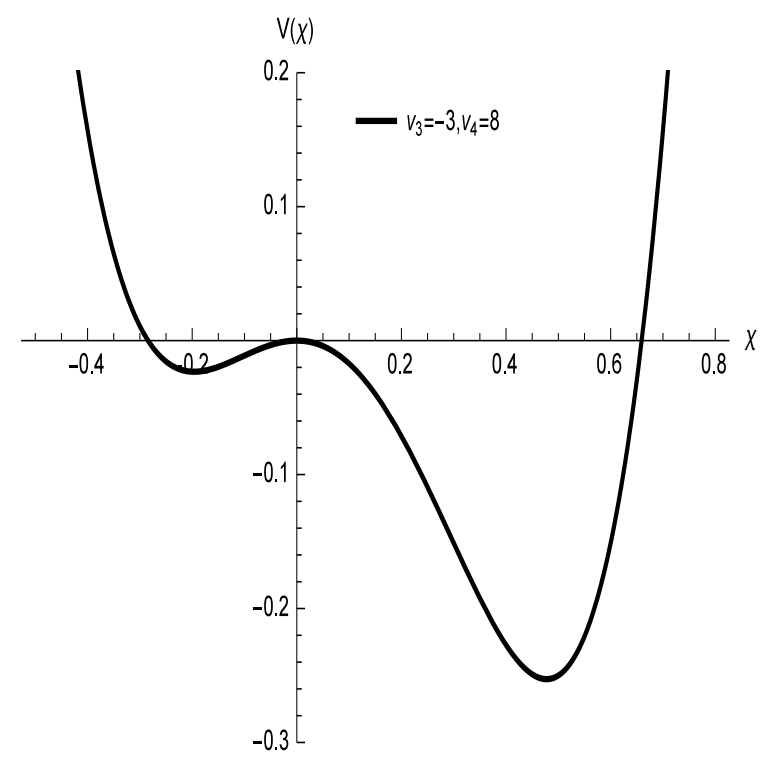

Figure 13. $V(\chi)$ as a function of $\chi$ with $v_{3}=-3, v_{4}=8$.

the left vacuum is around $\chi^{(-)}=-0.196$. From the potential level, we could see that the right vacuum is more stable due to the lower value of potential energy.

Inserting the potential into the equation of motion eq. (3.4), we solved the chiral condensate in chiral limit as shown in figure 14. From figure 14(a), we find that at low temperature there are still two branches of non-trivial solutions together with the trivial $\chi \equiv 0$ solution, while above $T=0.151 \mathrm{MeV}$ the non-trivial solutions disappears. Due to the cubic term, we could see that the positive(the red solid line plus the purple dashed line) and negative non-trivial branches(the blue dashed line) are no longer $\sigma \leftrightarrow-\sigma$ reflection of each other. As we read from figure 13, the potential energy of the right vacuum is lower. Therefore, we might expect that the positive sigma solutions would be more stable even in the chiral limit. To confirm this, we insert the $\chi(z)$ solution to the free energy formula eq. (3.5) and calculate the free energy density. The results are plotted in figure 14(b). From this plot, we do find that the free energy of the positive branch is smaller than the negative branch. As a result, the lower temperature region is dominated by the right vacuum. Then from the zooming out zone of figure 14(b), we find that the free energy density of the positive branch would start to be larger than zero, which shows that a phase transition would happen. The real physical path when increasing temperature would be the red solid line. The chiral condensate decrease from the finite vacuum value monotonously and at around $T=173 \mathrm{MeV}$ it suddenly drop to zero, which gives a first order picture of the chiral restoration phase transition. This chiral limit result is just the same as expected from the diagonal line in figure 1.

Now we turn the quark mass on. We find the low temperature $\chi \equiv 0$ would join the negative non-trivial branch to form a semi-circle again, while the high temperature $\chi \equiv 0$ branch would join the positive non-trivial branch and form a continuous line extend to high temperature. However, when $m_{q}$ is small, for example $m_{q}=7 \mathrm{MeV}$ as shown in the 


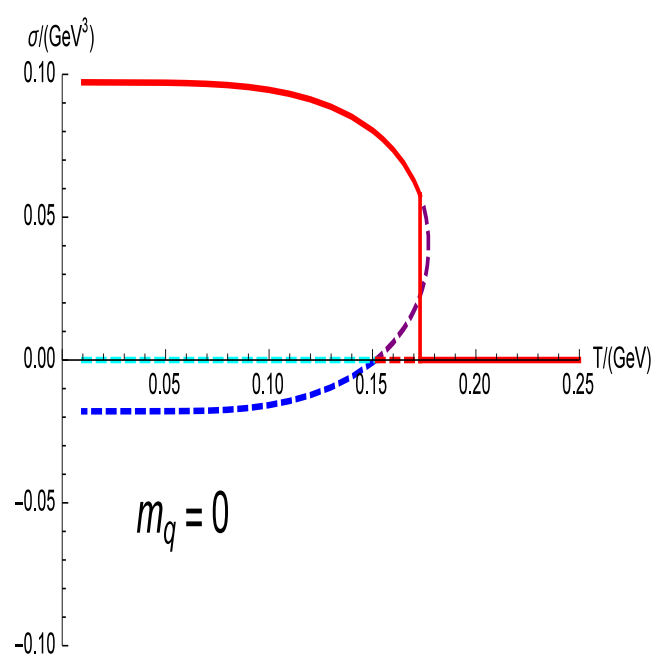

(a)

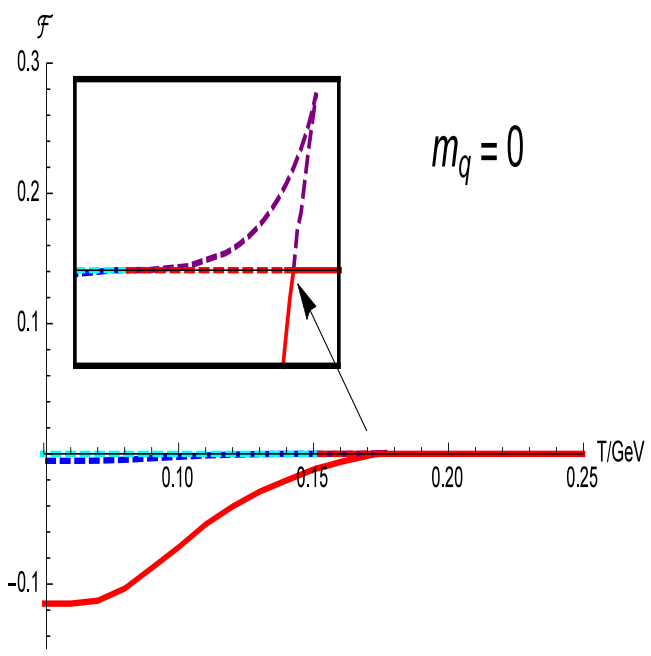

(b)

Figure 14. The results of chiral condensate $\sigma(T)$ in chiral limit and the corresponding free energy density $\mathcal{F}$ as a function of temperature $T$ in dilaton background eq. (4.1) when $v_{3}=-3, v_{4}=8$ and $\mu_{0}=0.43 \mathrm{GeV}, \mu_{1}=0.83 \mathrm{GeV}, \mu_{2}=0.176 \mathrm{GeV}$. Panel (a) shows the result of $\sigma(T)$ in chiral limit. Panel (b) compares the free energy density $\mathcal{F}$ of different solutions. The points representing the same solutions are labeled with the same colors and styles in panel (a) and panel (b).

dashed line of figure 15, the positive branch would be multivalued in the temperature range $167 \mathrm{MeV}<\mathrm{T}<175 \mathrm{MeV}$, which implies the first order property of the phase transition. In addition, when $m_{q}$ is large enough, for example $m_{q}=55 \mathrm{MeV}$ as shown in the dotdashed line in figure 15, the positive branch would decrease monotonously and continuously, which implies a crossover transition. In between these two types, it is not difficult to imagine that with certain value of $m_{q}$ the derivative $\frac{d \sigma}{d T}$ would be divergent at certain temperature, just as shown in the solid line in figure 15 (around $T=195 \mathrm{MeV}$ ). This mass would be the critical mass, which gives a second order phase transition. With the current parameter, we find that the critical mass is around $m_{q}=37 \mathrm{MeV}$.

In a short summary, when we turn on a negative cubic scalar potential, the model eq. (4.1) gives a first order chiral phase transition in chiral limit and in small quark mass region, while in sufficient large quark mass area, the phase transition would turn to be a crossover one. We summarize the results for $\mathrm{SU}(2)$ and $\mathrm{SU}(3)$ in figure 16. This agrees well with the picture shown in figure 1 (the top line and the diagonal line). Combining the results in $\mathrm{SU}(2)$ case and $\mathrm{SU}(3)$ case, we might expect that when extend to $N_{f}=2+1$ case, the model could produce phase diagram in mass plane similar to figure 1 qualitatively. We will leave this calculation in the future.

\section{Conclusion and discussion}

In this work, we investigate the chiral phase transition within the soft-wall holographic framework. By imposing proper UV and IR boundary conditions, we could solve chiral condensate as a function of quark mass and temperature. We first try in the original soft- 


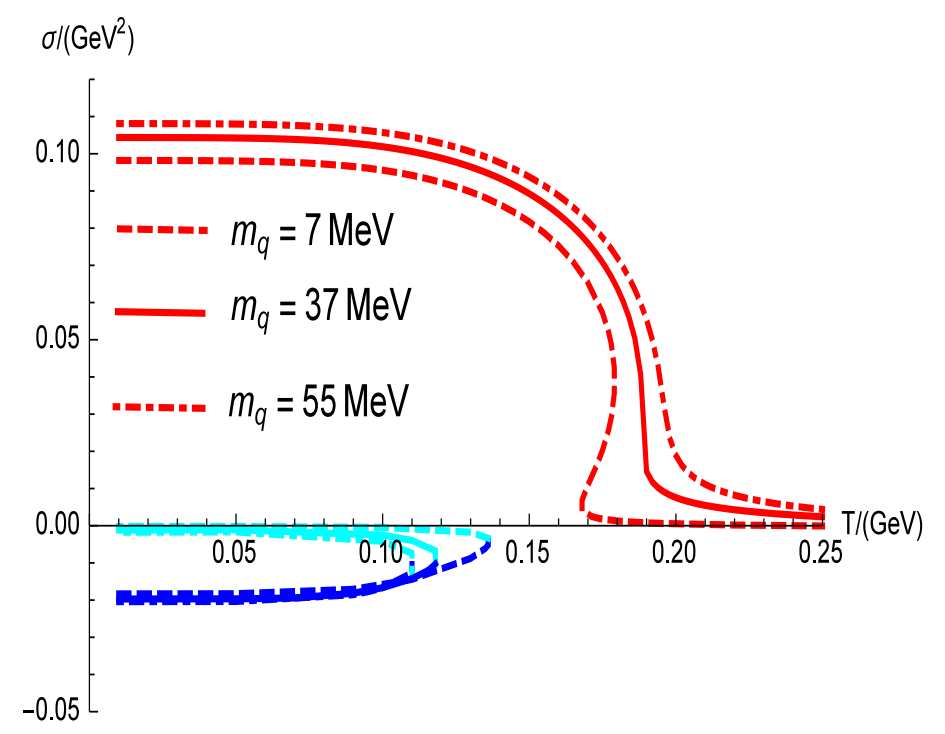

Figure 15. The results of chiral condensate $\sigma(T)$ as a function of temperature $T$ in dilaton background eq. (4.1) when $v_{3}=-3, v_{4}=8$ and $\mu_{0}=0.43 \mathrm{GeV}, \mu_{1}=0.83 \mathrm{GeV}, \mu_{2}=0.176 \mathrm{GeV}$. The red, cyan and blue dashed lines give the results of $m_{q}=7 \mathrm{MeV}$ and the solid and dotdashed lines give the results of $m_{q}=37 \mathrm{MeV}, \mathrm{m}_{\mathrm{q}}=55 \mathrm{MeV}$ respectively.

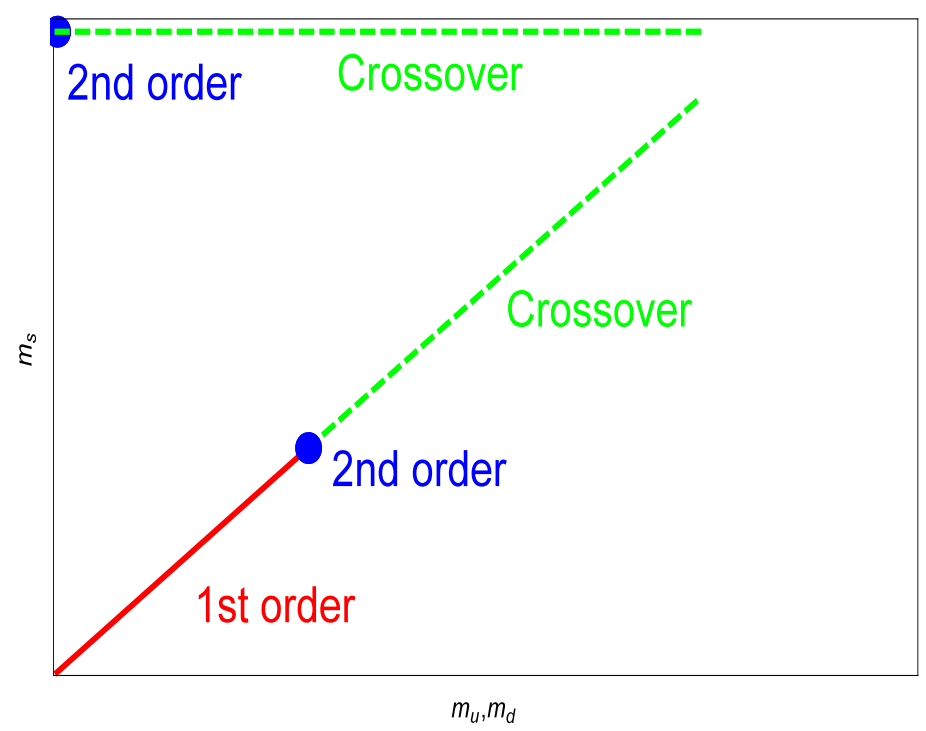

Figure 16. The $\mathrm{SU}(2)$ and $\mathrm{SU}(3)$ mass diagram in dilaton background eq. (4.1) when $v_{3}=-3, v_{4}=$ 8 and $\mu_{0}=0.43 \mathrm{GeV}, \mu_{1}=0.83 \mathrm{GeV}, \mu_{2}=0.176 \mathrm{GeV}$. The red solid line represents first order phase transition and the green dashed lines stand for crossover. The blue dots are second order points.

wall model with a pure positive quadratic dilaton and a mass term in the scalar potential. The results shown that in chiral limit there is no chiral condensate in the original soft-wall model, which implies that the chiral condensate in the original soft-wall model is induced by quark mass and the symmetry breaking is an explicit one. After introducing a quartic 
term in scalar potential to break the linear property of the equation of motion, we still find that in chiral limit the pure quadratic dilaton model can not realize the spontaneous chiral symmetry breaking.

Then, we consider the pure negative quadratic dilaton case with a positive quartic potential. We show that in this model, both the spontaneous chiral symmetry breaking in the vacuum and its restoration are realized correctly. The phase transition is a second order one in chiral limit and it turns to be a crossover one at any finite quark mass.

However, the negative part of dilaton field at large $z$ region would cause an massless scalar meson state, which has never been detected experimentally and is unacceptable. To cure this problem, we propose a dilaton model, which tends to be negative quadratic at small $z$ region and positive quadratic at large $z$ region. The intermediate interpolating configuration is controlled by two mass parameters. We find that in a large parameter region, the model can realize chiral phase transition well. In $\mathrm{SU}(2)$ case with positive value of the quartic scalar coefficient, it gives a second order phase transition in chiral limit and a crossover one at any finite quark mass. In $\mathrm{SU}(3)$ case with a negative cubic and positive quartic potential term, it gives a first order phase transition in chiral limit and small quark mass region, while in sufficient quark mass case, the phase transition turns out to be crossover again. Qualitatively, the results agrees perfectly with the Colombia sketch plot as shown in figure 1 . We also note that the interaction structures of the $5 \mathrm{D}$ soft-wall model discussed here is the same as the linear $\sigma$ model [5]. The $3+1$ dimension linear $\sigma$ model shows a first order phase transition with three or more massless flavors [5], which is similar to the results presented here. For two flavor case, it gives a second order phase transition when the coefficient of the determinant term is absent, which is also the same as our results. But in [5], the authors also consider the possibility of adding the determinant term in two flavor case, which could be a first order phase transition when properly tuning the temperature dependent coefficient of the determinant term. In our calculation, we have not considered the determinant term in two flavor case since in the $5 \mathrm{D}$ model the determinant term in two flavor case will change the coefficient of the mass term. Furthermore, quantitatively, we also show that if one take the proper value of the parameters, then the vacuum value of $\sigma$ would be around $(320 \mathrm{MeV})^{3}$ and the transition temperature is around $150 \mathrm{MeV}$, which are consistent with the lattice results. The studies here also show that the dominant scales for chiral dynamics and confinement are different, which is consistent with the previous studies in [85].

Finally, we would like to emphasize that we have not considered the meson spectra in a quantitative way. Instead, we only focus on the linear confinement, i.e. the Regge behavior in highly excited state. To generate meson spectra comparable to the experiment data, the simple interpolation selected in this work might be over simplified. We might need to modify the IR behavior of several quantities together. In general, such an IR modification could be imposing on metric, quartic term, dilaton and conformal mass as in $[23,69]$ to accommodate meson spectra simultaneously. Furthermore, we expect when extending to $N_{f}=2+1$ case, our model could generate the same qualitative results as shown in figure 1. We will leave these work to the future. 


\section{Acknowledgments}

The authors thank Song He, Yi Yang for valuable discussions. K.C is supported by CASTWAS president fellowship. M.H. is supported by the NSFC under Grant Nos. 11175251 and 11275213, DFG and NSFC (CRC 110), CAS key project KJCX2-EW-N01, and Youth Innovation Promotion Association of CAS. This work is funded in part by China Postdoctoral Science Foundation.

\section{A Numerical method}

In this section, we will describe how to determine chiral condensate numerically in the softwall model. Without loss of generality, we will take the simple negative dilaton $\Phi(z)=$ $-\mu^{2} z^{2}$ in the quartic potential model as an example. In this case, eq. (3.4) becomes

$$
\chi^{\prime \prime}-\left(\frac{3}{z}-2 \mu^{2} z+\frac{4 \pi^{4} T^{4} z^{3}}{1-\pi^{4} T^{4} z^{4}}\right) \chi^{\prime}+\frac{1}{z^{2}\left(1-\pi^{4} T^{4} z^{4}\right)}\left(3 \chi-4 v_{4} \chi^{3}\right)=0 .
$$

In principle, the above second order ordinary differential equation has two integral constants, which should be fixed by two boundary conditions. However, as easily can be read, there is an apparent singular point $z=z_{h}=1 /(\pi T)$ in the equation. This fact would lead one of the two solutions singular at this point. Therefore, if one requires the physical solution to be regular at the black hole horizon, then we can determine one of the two boundary coefficients from the other.

As mentioned in the previous sections, the boundary expansion of eq. (A.1) is of the following form

$$
\chi(z)=m_{q} \zeta z+\frac{\sigma}{\zeta} z^{3}+\ldots,
$$

where $m_{q}$ is mapped to the current quark mass and $\sigma$ is mapped to chiral condensate. As an example, here we take $m_{q}=0$. Isolating the apparent singular part of second order eqaution, we get $\frac{f^{\prime} \chi^{\prime}-e^{2 A_{s}} \partial_{\chi} V(\chi)}{f}$. Noting that to get a regular solution is equivalent to get a solution satisfying $\frac{f^{\prime} \chi^{\prime}-e^{2 A_{s}} \partial_{\chi} V(\chi)}{f}=0$, one can take the test function test $\equiv$ $-z^{2}\left(\frac{f^{\prime} \chi^{\prime}-e^{2 A_{s}} \partial_{\chi} V(\chi)}{f}\right)$ as a signal and tune $\sigma$ towards the correct value. In figure 17(a), we take $T=0.175 \mathrm{GeV}$ as an example and show this process explicitly. At the beginning, when we take $\sigma=0.050$, we see that at the horizon the test function blows up towards positive infinity (see the black dashed line). Then, we decrease $\sigma$ to 0.049 , and find that the test function blows up towards negative infinity (see the purple dashed line). This means that the physical value of $\sigma$ is between 0.050 and 0.049 . Therefore, we then try $\sigma$ to be the middle value of 0.050 and 0.049 . Repeating the process for several times, we find that when $\sigma=0.0494742 \ldots$, the test function can go smoothly through horizon (see the red dashed line), and we take this value as the physical value. In figure 17(b), we show that when $\sigma=0.0494742$, the numerical solution of $\chi$ can go smoothly through the horizon also. 


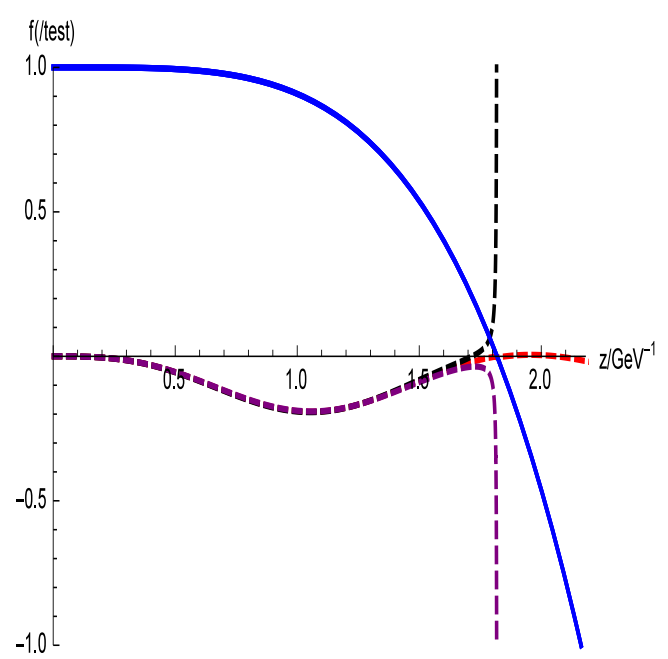

(a)

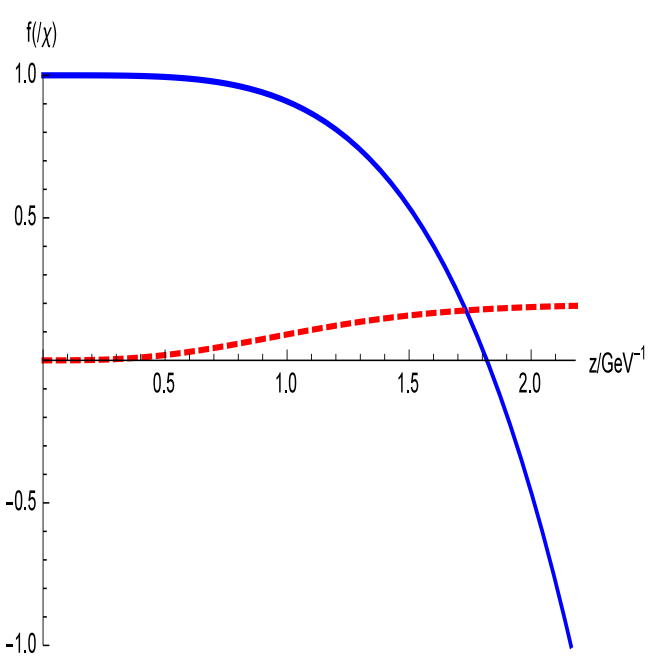

(b)

Figure 17. $\operatorname{test}(z)$ as a function of $z$ in negative quadratic dilaton background eq. (3.6) when $v_{3}=0, v_{4}=8$ and $m_{q}=0, \mu=1 \mathrm{GeV}, \mathrm{T}=0.175 \mathrm{GeV}$. Panel (a): the blue solid line represents $f(z)$ and the black, red, purple dashed lines represent for test $(z)$ solutions when $\sigma=0.050,0.0494742 \ldots, 0.049$ respectively. Panel (b): the blue solid line represents $f(z)$ and the red dashed line represents $\chi(z)$ when $\sigma=0.0494742$.

Open Access. This article is distributed under the terms of the Creative Commons Attribution License (CC-BY 4.0), which permits any use, distribution and reproduction in any medium, provided the original author(s) and source are credited.

\section{References}

[1] Y. Aoki, G. Endrodi, Z. Fodor, S.D. Katz and K.K. Szabo, The order of the quantum chromodynamics transition predicted by the standard model of particle physics, Nature 443 (2006) 675 [hep-lat/0611014] [INSPIRE].

[2] E. Laermann and O. Philipsen, The status of lattice QCD at finite temperature, Ann. Rev. Nucl. Part. Sci. 53 (2003) 163 [hep-ph/0303042] [INSPIRE].

[3] P. de Forcrand and O. Philipsen, The chiral critical line of $N(f)=2+1 Q C D$ at zero and non-zero baryon density, JHEP 01 (2007) 077 [hep-lat/0607017] [INSPIRE].

[4] K. Kanaya, Lattice results on the phase structure and equation of state in QCD at finite temperature, AIP Conf. Proc. 1343 (2011) 57 [arXiv: 1012.4235] [INSPIRE].

[5] R.D. Pisarski and F. Wilczek, Remarks on the Chiral Phase Transition in Chromodynamics, Phys. Rev. D 29 (1984) 338 [INSPIRE].

[6] J.M. Maldacena, The large- $N$ limit of superconformal field theories and supergravity, Int. J. Theor. Phys. 38 (1999) 1113 [hep-th/9711200] [INSPIRE].

[7] S.S. Gubser, I.R. Klebanov and A.M. Polyakov, Gauge theory correlators from noncritical string theory, Phys. Lett. B 428 (1998) 105 [hep-th/9802109] [InSPIRE].

[8] E. Witten, Anti-de Sitter space and holography, Adv. Theor. Math. Phys. 2 (1998) 253 [hep-th/9802150] [INSPIRE]. 
[9] J. Erlich, E. Katz, D.T. Son and M.A. Stephanov, QCD and a holographic model of hadrons, Phys. Rev. Lett. 95 (2005) 261602 [hep-ph/0501128] [INSPIRE].

[10] A. Karch, E. Katz, D.T. Son and M.A. Stephanov, Linear confinement and AdS/QCD, Phys. Rev. D 74 (2006) 015005 [hep-ph/0602229] [INSPIRE].

[11] G.F. de Teramond and S.J. Brodsky, Hadronic spectrum of a holographic dual of QCD, Phys. Rev. Lett. 94 (2005) 201601 [hep-th/0501022] [INSPIRE].

[12] L. Da Rold and A. Pomarol, Chiral symmetry breaking from five dimensional spaces, Nucl. Phys. B 721 (2005) 79 [hep-ph/0501218] [INSPIRE].

[13] J. Babington, J. Erdmenger, N.J. Evans, Z. Guralnik and I. Kirsch, Chiral symmetry breaking and pions in nonsupersymmetric gauge/gravity duals, Phys. Rev. D 69 (2004) 066007 [hep-th/0306018] [INSPIRE].

[14] M. Kruczenski, D. Mateos, R.C. Myers and D.J. Winters, Towards a holographic dual of large- $N_{c} Q C D$, JHEP 05 (2004) 041 [hep-th/0311270] [INSPIRE].

[15] T. Sakai and S. Sugimoto, Low energy hadron physics in holographic QCD, Prog. Theor. Phys. 113 (2005) 843 [hep-th/0412141] [INSPIRE].

[16] T. Sakai and S. Sugimoto, More on a holographic dual of QCD, Prog. Theor. Phys. 114 (2005) 1083 [hep-th/0507073] [INSPIRE].

[17] C. Csáki and M. Reece, Toward a systematic holographic QCD: A Braneless approach, JHEP 05 (2007) 062 [hep-ph/0608266] [INSPIRE].

[18] M. Huang, S. He, Q.-S. Yan and Y. Yang, Confront Holographic QCD with Regge Trajectories, Eur. Phys. J. C 66 (2010) 187 [arXiv:0710.0988] [INSPIRE].

[19] T. Gherghetta, J.I. Kapusta and T.M. Kelley, Chiral symmetry breaking in the soft-wall AdS/QCD model, Phys. Rev. D 79 (2009) 076003 [arXiv:0902.1998] [INSPIRE].

[20] T.M. Kelley, S.P. Bartz and J.I. Kapusta, Pseudoscalar Mass Spectrum in a Soft-Wall Model of AdS/QCD, Phys. Rev. D 83 (2011) 016002 [arXiv: 1009.3009] [inSPIRE].

[21] Y.-Q. Sui, Y.-L. Wu, Z.-F. Xie and Y.-B. Yang, Prediction for the Mass Spectra of Resonance Mesons in the Soft-Wall AdS/QCD with a Modified 5D Metric, Phys. Rev. D 81 (2010) 014024 [arXiv:0909.3887] [InSPIRE].

[22] Y.-Q. Sui, Y.-L. Wu and Y.-B. Yang, Predictive AdS/QCD Model for Mass Spectra of Mesons with Three Flavors, Phys. Rev. D 83 (2011) 065030 [arXiv:1012.3518] [INSPIRE].

[23] L.-X. Cui, Y.-L. Wu and Z. Fang, Infrared-improved soft-wall AdS/QCD model for mesons, Eur. Phys. J. C 76 (2016) 22 [arXiv:1310.6487] [InSPIRE].

[24] D. Li, M. Huang and Q.-S. Yan, A dynamical soft-wall holographic QCD model for chiral symmetry breaking and linear confinement, Eur. Phys. J. C 73 (2013) 2615 [arXiv: 1206.2824] [INSPIRE].

[25] D. Li and M. Huang, Dynamical holographic QCD model for glueball and light meson spectra, JHEP 11 (2013) 088 [arXiv: 1303.6929] [INSPIRE].

[26] P. Colangelo, F. De Fazio, F. Giannuzzi, F. Jugeau and S. Nicotri, Light scalar mesons in the soft-wall model of AdS/QCD, Phys. Rev. D 78 (2008) 055009 [arXiv:0807.1054] [InSPIRE].

[27] E.V. Shuryak, What RHIC experiments and theory tell us about properties of quark-gluon plasma?, Nucl. Phys. A 750 (2005) 64 [hep-ph/0405066] [INSPIRE].

[28] M.J. Tannenbaum, Recent results in relativistic heavy ion collisions: From 'a new state of matter' to 'the perfect fluid', Rept. Prog. Phys. 69 (2006) 2005 [nucl-ex/0603003] [InSPIRE]. 
[29] G. Policastro, D.T. Son and A.O. Starinets, The shear viscosity of strongly coupled $N=4$ supersymmetric Yang-Mills plasma, Phys. Rev. Lett. 87 (2001) 081601 [hep-th/0104066] [INSPIRE].

[30] R.-G. Cai, Z.-Y. Nie, N. Ohta and Y.-W. Sun, Shear Viscosity from Gauss-Bonnet Gravity with a Dilaton Coupling, Phys. Rev. D 79 (2009) 066004 [arXiv:0901.1421] [INSPIRE].

[31] R.-G. Cai, Z.-Y. Nie and Y.-W. Sun, Shear Viscosity from Effective Couplings of Gravitons, Phys. Rev. D 78 (2008) 126007 [arXiv:0811.1665] [INSPIRE].

[32] S.-J. Sin and I. Zahed, Holography of radiation and jet quenching, Phys. Lett. B 608 (2005) 265 [hep-th/0407215] [INSPIRE].

[33] E. Shuryak, S.-J. Sin and I. Zahed, A Gravity dual of RHIC collisions, J. Korean Phys. Soc. 50 (2007) 384 [hep-th/0511199] [INSPIRE].

[34] H. Nastase, The RHIC fireball as a dual black hole, hep-th/0501068 [INSPIRE].

[35] R.A. Janik and R.B. Peschanski, Asymptotic perfect fluid dynamics as a consequence of AdS/CFT, Phys. Rev. D 73 (2006) 045013 [hep-th/0512162] [INSPIRE].

[36] S. Nakamura and S.-J. Sin, A holographic dual of hydrodynamics, JHEP 09 (2006) 020 [hep-th/0607123] [INSPIRE].

[37] S.-J. Sin, S. Nakamura and S.P. Kim, Elliptic Flow, Kasner Universe and Holographic Dual of RHIC Fireball, JHEP 12 (2006) 075 [hep-th/0610113] [INSPIRE].

[38] C.P. Herzog, A. Karch, P. Kovtun, C. Kozcaz and L.G. Yaffe, Energy loss of a heavy quark moving through $N=4$ supersymmetric Yang-Mills plasma, JHEP 07 (2006) 013 [hep-th/0605158] [INSPIRE].

[39] S.S. Gubser, Drag force in AdS/CFT, Phys. Rev. D 74 (2006) 126005 [hep-th/0605182] [INSPIRE].

[40] Y. Wu, D. Hou and H.-c. Ren, Some Comments on the Holographic Heavy Quark Potential in a Thermal Bath, arXiv:1401.3635 [INSPIRE].

[41] D. Li, S. He and M. Huang, Temperature dependent transport coefficients in a dynamical holographic QCD model, JHEP 06 (2015) 046 [arXiv:1411.5332] [INSPIRE].

[42] D. Li, J. Liao and M. Huang, Enhancement of jet quenching around phase transition: result from the dynamical holographic model, Phys. Rev. D 89 (2014) 126006 [arXiv:1401.2035] [INSPIRE].

[43] O. Aharony, S.S. Gubser, J.M. Maldacena, H. Ooguri and Y. Oz, Large-N field theories, string theory and gravity, Phys. Rept. 323 (2000) 183 [hep-th/9905111] [INSPIRE].

[44] J. Erdmenger, N. Evans, I. Kirsch and E. Threlfall, Mesons in Gauge/Gravity Duals - A Review, Eur. Phys. J. A 35 (2008) 81 [arXiv:0711.4467] [inSPIRE].

[45] G.F. de Teramond and S.J. Brodsky, Hadronic Form Factor Models and Spectroscopy Within the Gauge/Gravity Correspondence, arXiv:1203.4025 [INSPIRE].

[46] Y. Kim, I.J. Shin and T. Tsukioka, Holographic QCD: Past, Present and Future, Prog. Part. Nucl. Phys. 68 (2013) 55 [arXiv:1205.4852] [InSPIRE].

[47] A. Adams, L.D. Carr, T. Schäfer, P. Steinberg and J.E. Thomas, Strongly Correlated Quantum Fluids: Ultracold Quantum Gases, Quantum Chromodynamic Plasmas and Holographic Duality, New J. Phys. 14 (2012) 115009 [arXiv:1205.5180] [InSPIRE].

[48] C.P. Herzog, A Holographic Prediction of the Deconfinement Temperature, Phys. Rev. Lett. 98 (2007) 091601 [hep-th/0608151] [INSPIRE]. 
[49] C.A. Ballon Bayona, H. Boschi-Filho, N.R.F. Braga and L.A. Pando Zayas, On a Holographic Model for Confinement/Deconfinement, Phys. Rev. D 77 (2008) 046002 [arXiv: 0705.1529] [INSPIRE].

[50] Y. Kim, B.-H. Lee, S. Nam, C. Park and S.-J. Sin, Deconfinement phase transition in holographic QCD with matter, Phys. Rev. D 76 (2007) 086003 [arXiv:0706. 2525] [INSPIRE].

[51] Y. Kim, T. Misumi and I.J. Shin, Holographic phase transition of QCD with back-reaction of flavors, arXiv:0911.3205 [INSPIRE].

[52] R.-G. Cai and J.P. Shock, Holographic confinement/deconfinement phase transitions of AdS/QCD in curved spaces, JHEP 08 (2007) 095 [arXiv:0705.3388] [INSPIRE].

[53] R.-G. Cai, S. Chakrabortty, S. He and L. Li, Some aspects of QGP phase in a hQCD model, JHEP 02 (2013) 068 [arXiv: 1209.4512] [INSPIRE].

[54] S.S. Gubser and A. Nellore, Mimicking the QCD equation of state with a dual black hole, Phys. Rev. D 78 (2008) 086007 [arXiv:0804.0434] [InSPIRE].

[55] S.S. Gubser, A. Nellore, S.S. Pufu and F.D. Rocha, Thermodynamics and bulk viscosity of approximate black hole duals to finite temperature quantum chromodynamics, Phys. Rev. Lett. 101 (2008) 131601 [arXiv:0804.1950] [INSPIRE].

[56] U. Gürsoy, E. Kiritsis, L. Mazzanti and F. Nitti, Deconfinement and Gluon Plasma Dynamics in Improved Holographic QCD, Phys. Rev. Lett. 101 (2008) 181601 [arXiv: 0804.0899] [INSPIRE].

[57] U. Gürsoy, E. Kiritsis, L. Mazzanti and F. Nitti, Holography and Thermodynamics of $5 D$ Dilaton-gravity, JHEP 05 (2009) 033 [arXiv: 0812.0792] [INSPIRE].

[58] O. Andreev, Renormalized Polyakov Loop in the Deconfined Phase of SU(N) Gauge Theory and Gauge/String Duality, Phys. Rev. Lett. 102 (2009) 212001 [arXiv:0903.4375] [INSPIRE].

[59] P. Colangelo, F. Giannuzzi and S. Nicotri, Holography, Heavy-Quark Free Energy and the QCD Phase Diagram, Phys. Rev. D 83 (2011) 035015 [arXiv: 1008.3116] [InSPIRE].

[60] D. Li, S. He, M. Huang and Q.-S. Yan, Thermodynamics of deformed AdS $S_{5}$ model with a positive/negative quadratic correction in graviton-dilaton system, JHEP 09 (2011) 041 [arXiv: 1103.5389] [INSPIRE].

[61] R.-G. Cai, S. He and D. Li, A hQCD model and its phase diagram in Einstein-Maxwell-Dilaton system, JHEP 03 (2012) 033 [arXiv:1201.0820] [INSPIRE].

[62] R. Yaresko and B. Kampfer, Equation of State and Viscosities from a Gravity Dual of the Gluon Plasma, Phys. Lett. B $\mathbf{7 4 7}$ (2015) 36 [arXiv:1306.0214] [INSPIRE].

[63] R. Yaresko, J. Knaute and B. Kämpfer, Cross-over versus first-order phase transition in holographic gravity-single-dilaton models of QCD thermodynamics, Eur. Phys. J. C 75 (2015) 295 [arXiv:1503.09065] [INSPIRE].

[64] J. Noronha, Polyakov Loops in Strongly-Coupled Plasmas with Gravity Duals, J. Phys. G 37 (2010) 094018 [arXiv:1001.3155] [InSPIRE].

[65] S.I. Finazzo and J. Noronha, Debye screening mass near deconfinement from holography, Phys. Rev. D 90 (2014) 115028 [arXiv:1411.4330] [InSPIRE].

[66] S. He, S.-Y. Wu, Y. Yang and P.-H. Yuan, Phase Structure in a Dynamical Soft-Wall Holographic QCD Model, JHEP 04 (2013) 093 [arXiv: 1301.0385] [INSPIRE].

[67] Y. Yang and P.-H. Yuan, A Refined Holographic QCD Model and QCD Phase Structure, JHEP 11 (2014) 149 [arXiv:1406.1865] [INSPIRE]. 
[68] Y. Yang and P.-H. Yuan, Confinement-deconfinement phase transition for heavy quarks in a soft wall holographic QCD model, JHEP 12 (2015) 161 [arXiv:1506.05930] [INSPIRE].

[69] L.-X. Cui, Z. Fang and Y.-L. Wu, Thermal Spectral Function and Deconfinement Temperature in Bulk Holographic AdS/QCD with Back Reaction of Bulk Vacuum, arXiv: 1404.0761 [INSPIRE].

[70] S.S. Afonin and A.D. Katanaeva, Holographic Estimates of the Deconfinement Temperature, Eur. Phys. J. C 74 (2014) 3124 [arXiv:1408.6935] [INSPIRE].

[71] F. Zuo, Thermal power terms in the Einstein-dilaton system, JHEP 06 (2014) 143 [arXiv: 1404.4512] [INSPIRE].

[72] F. Zuo and Y.-H. Gao, Quadratic thermal terms in the deconfined phase from holography, JHEP 07 (2014) 147 [arXiv: 1403.2241] [INSPIRE].

[73] Y. Aoki, Z. Fodor, S.D. Katz and K.K. Szabo, The QCD transition temperature: Results with physical masses in the continuum limit, Phys. Lett. B 643 (2006) 46 [hep-lat/0609068] [INSPIRE].

[74] Y. Aoki et al., The QCD transition temperature: results with physical masses in the continuum limit II., JHEP 06 (2009) 088 [arXiv:0903.4155] [INSPIRE].

[75] Y. Nambu and G. Jona-Lasinio, Dynamical model of elementary particles based on an analogy with superconductivity. I, Phys. Rev. 122 (1961) 345 [INSPIRE].

[76] Y. Nambu and G. Jona-Lasinio, Dynamical model of elementary particles based on an analogy with superconductivity. II, Phys. Rev. 124 (1961) 246 [INSPIRE].

[77] P. Colangelo, F. Giannuzzi, S. Nicotri and V. Tangorra, Temperature and quark density effects on the chiral condensate: An AdS/QCD study, Eur. Phys. J. C 72 (2012) 2096 [arXiv: 1112.4402] [INSPIRE].

[78] A. Barducci, R. Casalbuoni, G. Pettini and R. Gatto, Chiral phases of QCD at finite density and temperature, Phys. Rev. D 49 (1994) 426 [INSPIRE].

[79] K. Chelabi, Z. Fang, M. Huang, D. Li and Y.-L. Wu, Realization of chiral symmetry breaking and restoration in holographic QCD, arXiv:1511.02721 [INSPIRE].

[80] A. Cherman, T.D. Cohen and E.S. Werbos, The chiral condensate in holographic models of QCD, Phys. Rev. C 79 (2009) 045203 [arXiv:0804.1096] [inSPIRE].

[81] P. Breitenlohner and D.Z. Freedman, Positive Energy in anti-de Sitter Backgrounds and Gauged Extended Supergravity, Phys. Lett. B 115 (1982) 197 [InSPIRE].

[82] P. Breitenlohner and D.Z. Freedman, Stability in Gauged Extended Supergravity, Annals Phys. 144 (1982) 249 [inSPIRE].

[83] L. Mezincescu and P.K. Townsend, Stability at a Local Maximum in Higher Dimensional Anti-de Sitter Space and Applications to Supergravity, Annals Phys. 160 (1985) 406 [INSPIRE].

[84] A. Karch, E. Katz, D.T. Son and M.A. Stephanov, On the sign of the dilaton in the soft wall models, JHEP 04 (2011) 066 [arXiv: 1012.4813] [inSPIRE].

[85] E.V. Shuryak and T. Schäfer, The QCD vacuum as an instanton liquid, Ann. Rev. Nucl. Part. Sci. 47 (1997) 359 [INSPIRE]. 